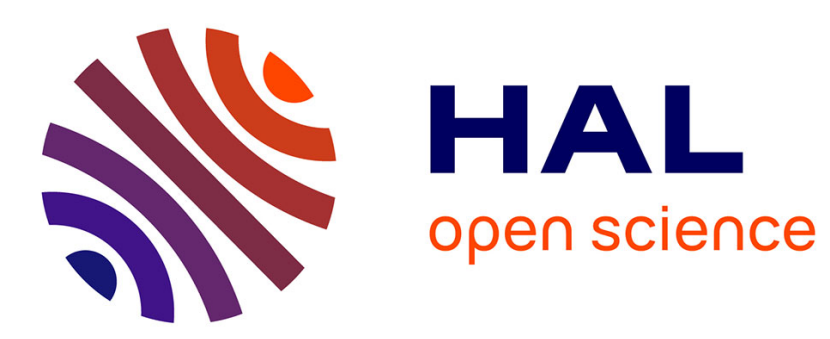

\title{
Null-controllability of two species reaction-diffusion system with nonlinear coupling: a new duality method
}

\author{
Kévin Le Balc'h
}

\section{To cite this version:}

Kévin Le Balc'h. Null-controllability of two species reaction-diffusion system with nonlinear coupling: a new duality method. SIAM Journal on Control and Optimization, 2019, 57 (4), pp.2541-2573. 10.1137/18M1173010 . hal-01717157

\section{HAL Id: hal-01717157 https://hal.science/hal-01717157}

Submitted on 26 Feb 2018

HAL is a multi-disciplinary open access archive for the deposit and dissemination of scientific research documents, whether they are published or not. The documents may come from teaching and research institutions in France or abroad, or from public or private research centers.
L'archive ouverte pluridisciplinaire HAL, est destinée au dépôt et à la diffusion de documents scientifiques de niveau recherche, publiés ou non, émanant des établissements d'enseignement et de recherche français ou étrangers, des laboratoires publics ou privés. 


\title{
NULL-CONTROLLABILITY OF TWO SPECIES REACTION-DIFFUSION SYSTEM WITH NONLINEAR COUPLING: A NEW DUALITY METHOD
}

\author{
KÉVIN LE BALC'H
}

\begin{abstract}
We consider a $2 \times 2$ nonlinear reaction-diffusion system posed on a smooth bounded domain $\Omega$ of $\mathbb{R}^{N}(N \geq 1)$. The control input is in the source term of only one equation. It is localized in some arbitrary nonempty open subset $\omega$ of the domain $\Omega$. First, we prove a global null-controllability result when the coupling term in the second equation is an odd power. As the linearized system around zero is not null-controllable, the usual strategy consists in using the return method, introduced by Jean-Michel Coron, or the method of power series expansions. In this paper, we give a direct nonlinear proof, which relies on a new duality method that we call Reflexive Uniqueness Method. It is a variation in reflexive Banach spaces of the well-known Hilbert Uniqueness Method, introduced by Jacques-Louis Lions. It is based on Carleman estimates in $L^{p}(2 \leq p<\infty)$ obtained from the usual Carleman inequality in $L^{2}$ and parabolic regularity arguments. This strategy enables us to find a control of the heat equation, which is an odd power of a regular function. Another advantage of the method is to produce small controls for small initial data. Secondly, thanks to the return method, we also prove a null-controllability result for more general nonlinear reaction-diffusion systems, where the coupling term in the second equation behaves as an odd power at zero.
\end{abstract}

\section{Contents}

1. Introduction

1.1. Context

1.2. Linearization

1.3. The "power system"

1.4. A direct approach

2. Main results

2.1. Definitions and usual properties

2.2. Main results

3. Global null-controllability for the "odd power system"

3.1. First step of the proof: steer $u$ to 0

3.2. Second step of the proof: steer $v$ to 0 thanks to a control which is as an odd power

3.3. Strategy of control in the whole interval $(0, T)$

4. A control for the heat equation which is an odd power

4.1. A Carleman inequality in $L^{2 k+2}$

4.2. A new penalized duality method in $L^{(2 k+2) /(2 k+1)}$, the Reflexive Uniqueness Method

4.3. A Bootstrap argument

5. Local null-controllability of general nonlinear systems

6. Some generalizations of the global null-controllability for "odd power systems"

6.1. Linear parabolic operators

6.2. Global null-controllability result for particular superlinearities $\quad 24$

6.3. Global null-controllability for all "power systems"

6.4. Global null-controllability for "even power systems" in $\mathbb{C}$ Appendix A. 


\section{INTRODUCTION}

Let $T>0, N \in \mathbb{N}^{*}, \Omega$ be a bounded, connected, open subset of $\mathbb{R}^{N}$ of class $C^{2}$, and let $\omega$ be a nonempty open subset of $\Omega$.

We consider a $2 \times 2$ nonlinear reaction-diffusion system with one internal control:

$$
\begin{cases}\partial_{t} u-\Delta u=f_{1}(u, v)+h 1_{\omega} & \text { in }(0, T) \times \Omega, \\ \partial_{t} v-\Delta v=f_{2}(u, v) & \text { in }(0, T) \times \Omega, \\ u, v=0 & \text { on }(0, T) \times \partial \Omega, \\ (u, v)(0, .)=\left(u_{0}, v_{0}\right) & \text { in } \Omega,\end{cases}
$$

with $f_{1}, f_{2} \in C^{\infty}\left(\mathbb{R}^{2} ; \mathbb{R}\right)$ satisfying $f_{1}(0,0)=f_{2}(0,0)=0$. Here, $(u, v)(t,):. \Omega \rightarrow \mathbb{R}^{2}$ is the state to be controlled, $h=h(t,):. \Omega \rightarrow \mathbb{R}$ is the control input supported in $\omega$.

We are interested in the null-controllability of (NL): for any initial data $\left(u_{0}, v_{0}\right)$, does there exist a control $h$ such that the solution $(u, v)$ of $(\mathrm{NL})$ verifies $(u, v)(T,)=$. $(0,0)$ ?

1.1. Context. The problem of null-controllability of the heat equation was solved independently by Gilles Lebeau, Luc Robbiano in 1995 (see [25] or the survey [24]) and Andrei Fursikov, Oleg Imanuvilov in 1996 (see [20]) with Carleman estimates.

Theorem 1.1. [3, Corollary 2]

For every $u_{0} \in L^{2}(\Omega)$, there exists $h \in L^{2}((0, T) \times \Omega)$ such that the solution $u$ of

$$
\begin{cases}\partial_{t} u-\Delta u=h 1_{\omega} & \text { in }(0, T) \times \Omega, \\ u=0 & \text { on }(0, T) \times \partial \Omega, \\ u(0, .)=u_{0} & \text { in } \Omega,\end{cases}
$$

satisfies $u(T,)=$.0 .

Then, null-controllability of linear and nonlinear coupled parabolic systems has been a challenging issue. For example, in [2], Farid Ammar-Khodja, Assia Benabdallah, Cédric Dupaix and Manuel Gonzalez-Burgos identified sharp conditions for the control of systems of the form

$$
\begin{cases}\partial_{t} U-D \Delta U=A U+B H 1_{\omega} & \text { in }(0, T) \times \Omega, \\ U=0 & \text { on }(0, T) \times \partial \Omega, \\ U(0, .)=U_{0} & \text { in } \Omega\end{cases}
$$

where $U(t,):. \Omega \rightarrow \mathbb{R}^{n}$ is the state, $H=H(t,):. \Omega \rightarrow \mathbb{R}^{m}$ is the control, $D:=$ $\operatorname{diag}\left(d_{1}, \ldots, d_{n}\right)$ with $d_{i} \in(0,+\infty)$ is the diffusion matrix, $A \in \mathcal{M}_{n}(\mathbb{R})$ (matrix with $n$ lines and $n$ columns with entries in $\mathbb{R}$ ) is the coupling matrix and $B \in \mathcal{M}_{n, m}(\mathbb{R})$ (matrix with $n$ lines and $m$ columns with entries in $\mathbb{R}$ ) represents the distribution of controls. In general, the rank of $B$ is less than $n$ (roughly speaking, there are less controls than equations), so that the controllability of the full system depends strongly on the (linear) coupling present in the system. We can see the survey [3] for other results (and open problems) on the controllability of linear coupled parabolic problems. The introduction of the article [23] provides an overview of the results on the controllability of linear and nonlinear coupled parabolic problems.

Roughly speaking, the null-controllability of (NL) can be reformulated as follows: how can the component $v$ be controlled thanks to the nonlinear coupling $f_{2}(u, v)$ ?

1.2. Linearization. We introduce the following notation which will be used throughout the paper,

$$
\forall \tau>0, Q_{\tau}=(0, \tau) \times \Omega .
$$

The usual strategy consists in proving a local null-controllability result for (NL) from a (global) null-controllability result for the linearized system of (NL) 
around $((\bar{u}, \bar{v}), \bar{h})=((0,0), 0)$. The linearized system $(\mathrm{L})$ is

$$
\begin{cases}\partial_{t} u-\Delta u=\frac{\partial f_{1}}{\partial u}(0,0) u+\frac{\partial f_{1}}{\partial v}(0,0) v+h 1_{\omega} & \text { in }(0, T) \times \Omega, \\ \partial_{t} v-\Delta v=\frac{\partial f_{2}}{\partial u}(0,0) u+\frac{\partial f_{2}}{\partial v}(0,0) v & \text { in }(0, T) \times \Omega, \\ u, v=0 & \text { on }(0, T) \times \partial \Omega, \\ (u, v)(0, .)=\left(u_{0}, v_{0}\right) & \text { in } \Omega .\end{cases}
$$

Definition 1.2. System (L) is said to be null-controllable if for every $\left(u_{0}, v_{0}\right) \in L^{2}(\Omega)^{2}$, there exists $h \in L^{2}\left(Q_{T}\right)$ such that the solution $(u, v)$ of $(\mathrm{L})$ satisfies $(u, v)(T,)=.(0,0)$.

Proposition 1.3. [3, Theorem 7.1]

The following statements are equivalent.

(1) System (L) is null-controllable.

(2) $\frac{\partial f_{2}}{\partial u}(0,0) \neq 0$.

Indeed, if $\frac{\partial f_{2}}{\partial u}(0,0)=0$, then the equation on $v$ is decoupled from the first equation of $(\mathrm{L})$. Consequently, for any initial data $\left(u_{0}, v_{0}\right) \in L^{2}(\Omega)^{2}$ such that $v_{0} \neq 0$, we have $v(T,) \neq$.0 by the backward uniqueness of the heat equation (see [5]). The proof of $(2) \Rightarrow(1)$ is a byproduct of Proposition 1.7.

Roughly speaking, $u$ can be driven to 0 thanks to the control $h$ and $v$ can be driven to 0 thanks to the coupling term $\frac{\partial f_{2}}{\partial u}(0,0) u$. We have the following diagram

$$
h \stackrel{\text { controls }}{\rightsquigarrow} u \stackrel{\text { controls }}{\rightsquigarrow} v \text {. }
$$

Definition 1.4. [Null-controllability]

(1) System (NL) is said to be locally null-controllable if there exists $\delta>0$ such that for every $\left(u_{0}, v_{0}\right) \in L^{\infty}(\Omega)^{2}$ satisfying $\left\|\left(u_{0}, v_{0}\right)\right\|_{L^{\infty}(\Omega)^{2}} \leq \delta$, there exists $h \in L^{2}\left(Q_{T}\right)$ such that $(\mathrm{NL})$ has a (unique) solution $(u, v) \in L^{\infty}\left(Q_{T}\right)^{2}$ that satisfies $(u, v)(T,)=.(0,0)$.

(2) System (NL) is said to be globally null-controllable if for every $\left(u_{0}, v_{0}\right) \in L^{\infty}(\Omega)^{2}$, there exists $h \in L^{2}\left(Q_{T}\right)$ such that (NL) has a (unique) solution $(u, v) \in L^{\infty}\left(Q_{T}\right)^{2}$ that satisfies $(u, v)(T,)=.(0,0)$.

Now, we mention the linear test for (NL) which is a corollary of Proposition 1.3.

Proposition 1.5. [11, Proof of Theorem 1]

Let us suppose that $\frac{\partial f_{2}}{\partial u}(0,0) \neq 0$. Then, (NL) is locally null-controllable.

Remark 1.6. This result is well-known but it is difficult to find in the literature (see [1, Theorem 6] with a restriction on the dimension $1 \leq N<6$ and other function spaces or one can adapt the arguments given in [11] to get Proposition 1.5 for any $N \in \mathbb{N}^{*}$ ). For other results in this direction, see [29], [26], [21] and [7].

The natural question is: what can we say about (NL) if the linearized system around $((0,0), 0)$ is not null-controllable i.e. when $\frac{\partial f_{2}}{\partial u}(0,0)=0$ ?

Another strategy to get local null-controllability for (NL) consists in linearizing around a non trivial trajectory $(\bar{u}, \bar{v}, \bar{h}) \in C^{\infty}\left(\overline{Q_{T}}\right)^{3}$ of the nonlinear system $(\mathrm{NL})$ which goes from 0 to 0 . This procedure is called the return method and was introduced by Jean-Michel Coron in [8] (see [9, Chapter 6]). The linearized system is the following one:

$($ L-bis $) \quad \begin{cases}\partial_{t} u-\Delta u=\frac{\partial f_{1}}{\partial u}(\bar{u}, \bar{v}) u+\frac{\partial f_{1}}{\partial v}(\bar{u}, \bar{v}) v+h 1_{\omega} & \text { in }(0, T) \times \Omega, \\ \partial_{t} v-\Delta v=\frac{\partial f_{2}}{\partial u}(\bar{u}, \bar{v}) u+\frac{\partial f_{2}}{\partial v}(\bar{u}, \bar{v}) v & \text { in }(0, T) \times \Omega, \\ u, v=0 & \text { on }(0, T) \times \partial \Omega, \\ (u, v)(0, .)=\left(u_{0}, v_{0}\right) & \text { in } \Omega .\end{cases}$ 
First, let us recall the generalization of Proposition 1.3 when the coupling coefficients are not constant. Historically, the proof is due to Luz de Teresa in [13].

Proposition 1.7. [3, Theorem 7.1]

We assume that there exist $t_{1}<t_{2} \in(0, T)$, a nonempty open subset $\omega_{0} \subset \omega$ and $\varepsilon>0$ such that $\left|\frac{\partial f_{2}}{\partial u}(\bar{u}(t, x), \bar{v}(t, x))\right| \geq \varepsilon$ for every $(t, x) \in\left(t_{1}, t_{2}\right) \times \omega_{0}$. Then, system (L-bis) is null-controllable (in the sense of Definition 1.2).

Then, the linear test gives the following result.

Proposition 1.8. [11, Proof of Theorem 1]

We assume that there exist $t_{1}<t_{2} \in(0, T)$, a nonempty open subset $\omega_{0} \subset \omega$ and $\varepsilon>0$ such that $\left|\frac{\partial f_{2}}{\partial u}(\bar{u}, \bar{v})\right| \geq \varepsilon$ on $\left(t_{1}, t_{2}\right) \times \omega_{0}$. Then, system (NL) is locally nullcontrollable.

Proposition 1.8 is used used in [11] with $f_{2}\left(u_{1}, u_{2}\right)=u_{1}^{3}+R u_{2}$, where $R \in \mathbb{R}$, [10], [12] and [23].

1.3. The "power system". A model-system for the question of null-controllability when the linearized system around $((0,0), 0)$ is not null-controllable is the following one:

(Power)

$$
\begin{cases}\partial_{t} u-\Delta u=h 1_{\omega} & \text { in }(0, T) \times \Omega, \\ \partial_{t} v-\Delta v=u^{n} & \text { in }(0, T) \times \Omega, \\ u, v=0 & \text { on }(0, T) \times \partial \Omega \\ (u, v)(0, .)=\left(u_{0}, v_{0}\right) & \text { in } \Omega\end{cases}
$$

where $n \geq 2$ is an integer.

Proposition 1.9. If $n$ is an even integer, then (Power) is not locally null-controllable.

Indeed, by the maximum principle $\left(u^{n} \geq 0\right)$, we have, for any solution of (Power) associated to an initial condition $\left(u_{0}, v_{0}\right)$ with $v_{0} \geq 0$ and $v_{0} \neq 0$,

$$
v(T, .) \geq \widetilde{v}(T, .) \geq 0 \quad \text { and } \quad \widetilde{v}(T, .) \neq 0,
$$

where $\widetilde{v}$ is the solution of the heat equation

$$
\begin{cases}\partial_{t} \widetilde{v}-\Delta \widetilde{v}=0 & \text { in }(0, T) \times \Omega, \\ \widetilde{v}=0 & \text { on }(0, T) \times \partial \Omega, \\ \widetilde{v}(0, .)=v_{0} & \text { in } \Omega .\end{cases}
$$

The following result is due to Jean-Michel Coron, Sergio Guerrero and Lionel Rosier. The proof is based on the return method (see [11]).

Proposition 1.10. [11, Theorem 1]

If $n=3$, then (Power) is locally null-controllable.

Remark 1.11. The difficult point of the proof of Proposition 1.10 is the construction of the nontrivial trajectory (see [11, Section 2]). The method can be generalized to $n=2 k+1$ for $k \in \mathbb{N}^{*}$ but with longer computations. The same problem appears in [31, Section 4.2].

Remark 1.12. An homogeneity argument shows that for the system (Power) the local null-controllability implies the global null-controllability (consider $u_{\varepsilon}=\varepsilon u$, $\left.v_{\varepsilon}=\varepsilon^{n} v, h_{\varepsilon}=\varepsilon h\right)$. However, this strategy does not provide estimate on the control. This kind of argument is used in [12]. In this paper, we propose a different direct method for the global null-controllability, that provides estimates. 
1.4. A direct approach. From now on, $k \in \mathbb{N}^{*}$ is fixed.

The first goal of this paper is to give a direct proof (i.e. without return method) of the global null-controllability of the system

$$
\begin{cases}\partial_{t} u-\Delta u=h 1_{\omega} & \text { in }(0, T) \times \Omega \\ \partial_{t} v-\Delta v=u^{2 k+1} & \text { in }(0, T) \times \Omega \\ u, v=0 & \text { on }(0, T) \times \partial \Omega \\ (u, v)(0, .)=\left(u_{0}, v_{0}\right) & \text { in } \Omega\end{cases}
$$

Our proof is based on a new duality method, called Reflexive Uniqueness Method. The first step will consist in proving a Carleman estimate in $L^{2 k+2}$ for the heat equation (see Section 4.1 and particularly Theorem 4.4). The second step will consist in considering a penalized problem in $L^{\frac{2 k+2}{2 k+1}}$ (see Section 4.2), a generalization of the Penalized Hilbert Uniqueness Method, introduced by Jacques-Louis Lions (see [27] and also [32, Section 2] for an introduction to the Hilbert Uniqueness Method and some generalizations). This procedure enables us to find a control of the heat equation which is an odd power of a regular function.

The second goal of this paper is to prove a local null-controllability result for more general systems than (Odd) thanks to the return method (introduced in Section 1).

\section{MAIN RESUltS}

\subsection{Definitions and usual properties.}

Definition 2.1. We introduce the functional space

$$
W_{T}:=L^{2}\left(0, T ; H_{0}^{1}(\Omega)\right) \cap H^{1}\left(0, T ; H^{-1}(\Omega)\right) .
$$

Proposition 2.2. [16, Section 5.9.2, Theorem 3]

We have the embedding

$$
W_{T} \hookrightarrow C\left([0, T] ; L^{2}(\Omega)\right) .
$$

We have this well-posedness result for linear parabolic systems.

Definition-Proposition 2.3. Let $l \in \mathbb{N}^{*}, y_{0} \in L^{2}(\Omega)^{l}, g \in L^{2}\left(Q_{T}\right)^{l}$. The following Cauchy problem admits a unique weak solution $y \in W_{T}^{l}$

$$
\begin{cases}\partial_{t} y-\Delta y=g & \text { in }(0, T) \times \Omega, \\ y=0 & \text { on }(0, T) \times \partial \Omega, \\ y(0, .)=y_{0} & \text { in } \Omega .\end{cases}
$$

This means that $y$ is the unique function in $W_{T}^{l}$ which satisfies the variational formulation

(4) $\forall w \in L^{2}\left(0, T ; H_{0}^{1}(\Omega)^{l}\right), \int_{0}^{T}\left(\partial_{t} y, w\right)_{\left.\left(H^{-1}(\Omega)^{l}\right), H_{0}^{1}(\Omega)^{l}\right)}+\int_{Q_{T}} \nabla y \cdot \nabla w=\int_{Q_{T}} g \cdot w$,

and

$$
y(0, .)=y_{0} \text { in } L^{2}(\Omega)^{l} .
$$

Moreover, there exists $C>0$ independent of $y_{0}$ and $g$ such that

$$
\|y\|_{W_{T}^{l}} \leq C\left(\left\|y_{0}\right\|_{L^{2}(\Omega)^{l}}+\|g\|_{L^{2}\left(Q_{T}\right)^{l}}\right) .
$$

Let $p \in[1,+\infty], y_{0} \in L^{p}(\Omega)^{l}$ and $g \in L^{p}\left(Q_{T}\right)^{l}$, then $y \in L^{p}\left(Q_{T}\right)^{l}$ and there exists $C>0$ independent of $y_{0}$ and $g$ such that

$$
\|y\|_{L^{p}\left(Q_{T}\right)^{l}} \leq C\left(\left\|y_{0}\right\|_{L^{p}(\Omega)^{l}}+\|g\|_{L^{p}\left(Q_{T}\right)^{l}}\right) .
$$


Proof. First, the well-posedness in $W_{T}^{l}$ (i.e. (4), (5) and (6)) is based on Galerkin approximations and energy estimates. One can easily adapt the arguments given in [16, Section 7.1.2].

Secondly, the $L^{p}$-estimate (i.e. (7) for $p<+\infty$ ) is based on the application of (4) with a cut-off of $w=|y|^{p-2} y$.

Finally, the $L^{\infty}$-estimate (i.e. (7) for $p=+\infty$ ) is based on Stampacchia's method (see the proof of [22, Chapter 3, Paragraph 7, Theorem 7.1]).

The following definition-proposition justifies the notion of (unique) solution associated to a control and therefore, the definition of local null-controllability and global null-controllability (already introduced in Section 1, see Definition 1.4).

Definition-Proposition 2.4. Let $\left(u_{0}, v_{0}\right) \in L^{\infty}(\Omega)^{2}, h \in L^{2}\left(Q_{T}\right)$.

Let $(u, v) \in\left(W_{T} \cap L^{\infty}\left(Q_{T}\right)\right)^{2}$. We say that $(u, v)$ is a solution of (NL) if $(u, v)$ satisfies

$$
\begin{aligned}
& \forall\left(w_{1}, w_{2}\right) \in L^{2}\left(0, T ; H_{0}^{1}(\Omega)\right)^{2} \\
& \int_{0}^{T}\left(\partial_{t} u, w_{1}\right)_{\left(H^{-1}(\Omega), H_{0}^{1}(\Omega)\right)}+\int_{Q_{T}} \nabla u . \nabla w_{1}=\int_{Q_{T}}\left(f_{1}(u, v)+h 1_{\omega}\right) w_{1}, \\
& \int_{0}^{T}\left(\partial_{t} v, w_{2}\right)_{\left(H^{-1}(\Omega), H_{0}^{1}(\Omega)\right)}+\int_{Q_{T}} \nabla v \cdot \nabla w_{2}=\int_{Q_{T}} f_{2}(u, v) w_{2},
\end{aligned}
$$

and

$$
(u, v)(0, .)=\left(u_{0}, v_{0}\right) \text { in } L^{\infty}(\Omega)^{2} .
$$

Let $(u, v) \in\left(W_{T} \cap L^{\infty}\left(Q_{T}\right)\right)^{2}$ and $(\widetilde{u}, \widetilde{v}) \in\left(W_{T} \cap L^{\infty}\left(Q_{T}\right)\right)^{2}$ be two solutions of (NL). Then, $(u, v)=(\widetilde{u}, \widetilde{v})$.

Proof. The nonlinearities $f_{1}$ and $f_{2}$ are in $C^{\infty}\left(\mathbb{R}^{2}, \mathbb{R}\right)$, thus they are locally Lipschitz on $\mathbb{R}^{2}$. This provides the uniqueness of the solution of $(\mathrm{NL})$ in $L^{\infty}\left(Q_{T}\right)$ (associated to an initial data $\left(u_{0}, v_{0}\right) \in L^{\infty}(\Omega)^{2}$ and a control $h \in L^{2}\left(Q_{T}\right)$ ) by a Gronwall argument (see Appendix A.1).

2.2. Main results. Our first main result is the following one.

Theorem 2.5. The system (Odd) is globally null-controllable (in the sense of Definition 1.4).

More precisely, there exists $\left(C_{p}\right)_{p \in[2,+\infty)} \in(0, \infty)^{[2,+\infty)}$ such that for every initial data $\left(u_{0}, v_{0}\right) \in L^{\infty}(\Omega)^{2}$, there exists a control $h \in \bigcap_{p \in[2,+\infty)} L^{p}\left(Q_{T}\right)$ satisfying

$$
\forall p \in[2,+\infty),\|h\|_{L^{p}\left(Q_{T}\right)} \leq C_{p}\left(\left\|u_{0}\right\|_{L^{\infty}(\Omega)}+\left\|v_{0}\right\|_{L^{\infty}(\Omega)}^{1 /(2 k+1)}\right),
$$

and the solution $(u, v)$ of (Odd) verifies

$$
(u, v)(T, .)=(0,0) .
$$

Remark 2.6. We give some natural extensions of this result in Section 6 .

Remark 2.7. If we assume that

$$
\Omega \in C^{2, \alpha},
$$

with $0<\alpha<1$, then Theorem 2.5 remains true with a control $h \in L^{\infty}\left(Q_{T}\right)$ and the estimate (11) holds true with $p=+\infty$ (see Remark 3.9 and Remark 4.11).

Our second main result is a local controllability result for more general reactiondiffusion systems than (Odd). 
Theorem 2.8. Let $\left(g_{1}, g_{2}\right) \in C^{\infty}(\mathbb{R} ; \mathbb{R})^{2}$ be such that

$$
\begin{aligned}
& g_{1}(0)=g_{1}^{\prime}(0)=\cdots=g_{1}^{(2 k)}(0)=0 \text { and } g_{1}^{(2 k+1)}(0) \neq 0, \\
& g_{2}(0) \neq 0 .
\end{aligned}
$$

Let $f_{1} \in C^{\infty}\left(\mathbb{R}^{2} ; \mathbb{R}\right), f_{2} \in C^{\infty}\left(\mathbb{R}^{2} ; \mathbb{R}\right)$ be such that

$$
\begin{aligned}
& \forall v \in \mathbb{R}, f_{1}(0, v)=0, \\
& \forall(u, v) \in \mathbb{R}^{2}, f_{2}(u, v):=g_{1}(u) g_{2}(v) .
\end{aligned}
$$

Then, the system (NL) is locally null-controllable (in the sense of Definition 1.4).

Application 2.9. By taking $f_{1}(u, v)=-u^{2 k+1}=-f_{2}(u, v)$, Theorem 2.8 shows the local null-controllability of a model for the non reversible chemical reaction (according to the law of mass action and the Fick's law)

$$
(2 k+1) U \rightarrow V,
$$

where $u$ and $v$ denote respectively the concentrations of the component $U$ and $V$.

However, we can't deduce from Theorem 2.8 a local null-controllability result (which is true for $k=1$ thanks to [11, Theorem 1]) of a model for the reversible chemical reaction

$$
(2 k+1) U \rightleftharpoons V,
$$

which corresponds to $f_{1}(u, v)=-u^{2 k+1}+v=-f_{2}(u, v)$.

By taking $f_{1}(u, v)=\left(k_{2}-\left(2 k_{1}+1\right)\right) u^{2 k_{1}+1}+\left(k_{5}-\left(2 k_{1}+1\right)\right) u^{2 k_{1}+1} v^{k_{4}}$ and $f_{2}(u, v)=$ $k_{3} u^{2 k_{1}+1}+\left(k_{6}-k_{4}\right) u^{2 k_{1}+1} v^{k_{4}}$ with $k_{1}, k_{2}, k_{3}, k_{4}, k_{5}, k_{6}$ positive integers, Theorem 2.8 shows the local null-controllability of a model for the chemical reaction

$$
\begin{cases}\left(2 k_{1}+1\right) U & \rightarrow k_{2} U+k_{3} V, \\ \left(2 k_{1}+1\right) U+k_{4} V & \rightarrow k_{5} U+k_{6} V .\end{cases}
$$

From now on, unless otherwise specified, we denote by $C$ (respectively $C_{r}$ ) a positive constant (respectively a positive constant which depends on the parameter r) that may change from line to line.

\section{Global null-CONTROllability FOR the "OdD POWer System"}

The aim of this part is to prove Theorem 2.5. We now fix $\left(u_{0}, v_{0}\right) \in L^{\infty}(\Omega)^{2}$ until the end of the section.

3.1. First step of the proof: steer $u$ to 0 . First, we find a control of (Odd) which steers $u$ to 0 in time $T / 2$.

Proposition 3.1. There exists $h_{1} \in L^{\infty}((0, T / 2) \times \Omega)$ satisfying

$$
\left\|h_{1}\right\|_{L^{\infty}((0, T / 2) \times \Omega)} \leq C\left\|u_{0}\right\|_{L^{\infty}(\Omega)},
$$

such that the solution $\left(u_{1}, v_{1}\right) \in L^{\infty}((0, T / 2) \times \Omega)^{2}$ of

$$
\begin{cases}\partial_{t} u_{1}-\Delta u_{1}=h_{1} 1_{\omega} & \text { in }(0, T / 2) \times \Omega, \\ \partial_{t} v_{1}-\Delta v_{1}=u_{1}^{2 k+1} & \text { in }(0, T / 2) \times \Omega, \\ u_{1}, v_{1}=0 & \text { on }(0, T / 2) \times \partial \Omega, \\ \left(u_{1}, v_{1}\right)(0, .)=\left(u_{0}, v_{0}\right) & \text { in } \Omega,\end{cases}
$$

satisfies $u_{1}(T / 2,)=$.0 . Moreover, we have

$$
\left\|v_{1}(T / 2, .)\right\|_{L^{\infty}(\Omega)} \leq C\left(\left\|u_{0}\right\|_{L^{\infty}(\Omega)}^{2 k+1}+\left\|v_{0}\right\|_{L^{\infty}(\Omega)}\right) .
$$

Proof. The proof is based on the following result (see Remark 3.3 for some references). 
Proposition 3.2. [Null-controllability in $L^{\infty}$ of the linear heat equation in any time] For every $\tau>0, y_{0} \in L^{\infty}(\Omega)$, there exists $h_{\tau} \in L^{\infty}\left(Q_{\tau}\right)$ satisfying

$$
\|h\|_{L^{\infty}((0, \tau) \times \Omega)} \leq C_{\tau}\left\|y_{0}\right\|_{L^{\infty}(\Omega)},
$$

such that the solution $y \in L^{\infty}\left(Q_{\tau}\right)$ of

$$
\begin{cases}\partial_{t} y-\Delta y=h_{\tau} 1_{\omega} & \text { in }(0, \tau) \times \Omega, \\ y=0 & \text { on }(0, \tau) \times \partial \Omega, \\ y(0, .)=y_{0} & \text { in } \Omega,\end{cases}
$$

satisfies $y(\tau,)=$.0 .

We use Proposition 3.2 by taking $\tau=T / 2, y_{0}=u_{0}$. We get the existence of a control $h_{1} \in L^{\infty}((0, T / 2) \times \Omega)$ satisfying (15) which steers $u_{1} \in L^{\infty}((0, T / 2) \times \Omega)$ to 0 :

$$
\begin{cases}\partial_{t} u_{1}-\Delta u_{1}=h_{1} 1_{\omega} & \text { in }(0, \tau) \times \Omega, \\ u_{1}=0 & \text { on }(0, \tau) \times \partial \Omega, \\ \left(u_{1}(0, .), u_{1}(T / 2, .)\right)=\left(u_{0}, 0\right) & \text { in } \Omega .\end{cases}
$$

Moreover, from (19), (7) with $p=+\infty$ and (15), we get

$$
\left\|u_{1}\right\|_{L^{\infty}((0, T / 2) \times \Omega)} \leq C\left\|u_{0}\right\|_{L^{\infty}(\Omega)} .
$$

Then, we set $v_{1} \in L^{\infty}((0, T / 2) \times \Omega)$ (see Definition-Proposition 2.3 with $p=+\infty$ ), as the solution of

$$
\begin{cases}\partial_{t} v_{1}-\Delta v_{1}=u_{1}^{2 k+1} & \text { in }(0, T / 2) \times \Omega, \\ v_{1}=0 & \text { on }(0, T / 2) \times \partial \Omega, \\ v_{1}(0, .)=v_{0} & \text { in } \Omega .\end{cases}
$$

From (21), (7) with $p=+\infty$ and (20), we have (16).

Remark 3.3. There exists at least three proofs of Proposition 3.2. First, the common argument is the null-controllability of the heat equation in $L^{2}$ proved independently by Gilles Lebeau, Luc Robbiano in 1995 (see [25] and [24]) and Andrei Fursikov, Oleg Imanuvilov in 1996 (see [20]). Then, the goal is to get a control in $L^{\infty}$. The first method has been employed for the first time by Enrique Fernandez-Cara and Enrique Zuazua (see [19, Theorem 3.1]) and it is based on the local regularizing effect of the heat equation which leads to a refined observability inequality (see [19, Proposition 3.2]). The second method has been employed for the first time by Viorel Barbu (see [4]) and it is based on a Penalized Hilbert Uniqueness Method (see also [11, Section 3.1.2]). The more recent method is due to Olivier Bodart, Manuel Gonzalez-Burgos, Rosario Pérez-Garcia (see [6]) and it is sometimes called the fictitious control method (see [17, Section 2] for the Neumann case and [15]).

3.2. Second step of the proof: steer $v$ to 0 thanks to a control which is as an odd power. The aim of this part is to find a control of (Odd) which steers $v$ to 0 (and $u$ from 0 to 0 ) in time $T$.

Proposition 3.4. Let $\left(\left(u_{1}, v_{1}\right), h_{1}\right)$ as in Proposition 3.1.

There exists a control $h_{2} \in \bigcap_{p \in[2,+\infty)} L^{p}((T / 2, T) \times \Omega)$ satisfying

$$
\forall p \in[2,+\infty),\left\|h_{2}\right\|_{L^{p}((T / 2, T) \times \Omega)} \leq C_{p}\left(\left\|u_{0}\right\|_{L^{\infty}(\Omega)}+\left\|v_{0}\right\|_{L^{\infty}(\Omega)}^{1 /(2 k+1)}\right),
$$

such that the solution $\left(u_{2}, v_{2}\right) \in L^{\infty}((T / 2, T) \times \Omega)^{2}$ of

$$
\begin{cases}\partial_{t} u_{2}-\Delta u_{2}=h_{2} 1_{\omega} & \text { in }(T / 2, T) \times \Omega, \\ \partial_{t} v_{2}-\Delta v_{2}=u_{2}^{2 k+1} & \text { in }(T / 2, T) \times \Omega, \\ u_{2}, v_{2}=0 & \text { on }(T / 2, T) \times \partial \Omega \\ \left(u_{2}, v_{2}\right)(T / 2, .)=\left(0, v_{1}(T / 2, .)\right) & \text { in } \Omega,\end{cases}
$$

satisfies $\left(u_{2}, v_{2}\right)(T,)=.(0,0)$. 
Our approach consists in looking at the second equation of (Odd) like a controlled heat equation where the state is $v(t,$.$) and the control input is u^{2 k+1}(t,$.$) . Here, the$ question consists in proving that the heat equation is null-controllable with localized control which is as an odd power of a regular function.

For the sequel, we need to introduce some usual definitions and properties.

Definition 3.5. The mapping $x \in \mathbb{R} \mapsto x^{2 k+1} \in \mathbb{R}$ is one-to-one. We note its inverse function $x \mapsto x^{\frac{1}{2 k+1}}$.

Definition 3.6. For all $\tau>0,0<\tau_{1}<\tau_{2}, p \in[1,+\infty]$, we introduce the functional spaces

$$
\begin{gathered}
X_{\tau, p}=L^{p}\left(0, \tau ; W^{2, p} \cap W_{0}^{1, p}(\Omega)\right) \cap W^{1, p}\left(0, \tau ; L^{p}(\Omega)\right), \\
X_{\left(\tau_{1}, \tau_{2}\right), p}=L^{p}\left(\tau_{1}, \tau_{2} ; W^{2, p} \cap W_{0}^{1, p}(\Omega)\right) \cap W^{1, p}\left(\tau_{1}, \tau_{2} ; L^{p}(\Omega)\right),
\end{gathered}
$$

The following result is new and it is the key point of this section.

Proposition 3.7. For every $\tau>0$, there exists $C_{\tau}>0$ such that for every $y_{0} \in L^{\infty}(\Omega)$, there exists a control $h_{\tau} \in L^{\infty}\left(Q_{\tau}\right)$ which verifies

$$
\begin{aligned}
& \left\|h_{\tau}^{\frac{1}{2 k+1}}\right\|_{L^{\infty}\left(Q_{\tau}\right)} \leq C_{\tau}\left\|y_{0}\right\|_{L^{\infty}(\Omega)}^{1 /(2 k+1)}, \\
& h_{\tau}^{\frac{1}{2 k+1}} \in \bigcap_{p \in[2,+\infty)} X_{\tau, p}, \\
& \forall p \in[2,+\infty), \exists C_{\tau, p}>0,\left\|h_{\tau}^{\frac{1}{2 k+1}}\right\|_{X_{\tau, p}} \leq C_{\tau, p}\left\|y_{0}\right\|_{L^{\infty}(\Omega)}^{1 /(2 k+1)}, \\
& h_{\tau}(0, .)=h_{\tau}(\tau, .)=0, \\
& \forall t \in[0, \tau], \operatorname{supp}\left(h_{\tau}(t, .)\right) \subset \subset \omega,
\end{aligned}
$$

such that the solution $y \in L^{\infty}\left(Q_{\tau}\right)$ of (18) satisfies $y(\tau,)=$.0 .

Remark 3.8. Proposition 3.7 extends Proposition 3.2. Its proof is inspired by the Penalized Hilbert Uniqueness Method introduced by Barbu (see [4]). It is based on the Reflexive Uniqueness Method.

Remark 3.9. If we assume that (12) holds true, then we can replace $\bigcap_{p \in[2,+\infty)} X_{\tau, p}$ by $C^{1,2}\left(\overline{Q_{T}}\right)$. It easily gives the proof of Remark 2.7 by adapting the proof of Proposition 3.4.

Before proving this proposition (whose proof is reported to Section 4), we apply it to our problem.

Proof. We apply Proposition 3.7 with $(0, \tau) \leftarrow(T / 2, T), y_{0} \leftarrow v_{1}(T / 2,.) \in L^{\infty}(\Omega)$. Then, there exists a control $H \in L^{\infty}((T / 2, T) \times \Omega)$ such that

$$
\begin{aligned}
& H^{\frac{1}{2 k+1}} \in \bigcap_{p \in[2,+\infty)} X_{(T / 2, T), p}, \\
& \forall p \in[2,+\infty),\left\|H^{\frac{1}{2 k+1}}\right\|_{X_{(T / 2, T), p}} \leq C_{p}\left\|v_{1}(T / 2, .)\right\|_{L^{\infty}(\Omega)}^{1 /(2 k+1)}, \\
& H(T / 2, .)=H(T, .)=0, \\
& \forall t \in[T / 2, T], \operatorname{supp}(H(t, .)) \subset \subset \omega,
\end{aligned}
$$

and the solution $v_{2}$ of

$$
\begin{cases}\partial_{t} v_{2}-\Delta v_{2}=H & \text { in }(T / 2, T) \times \Omega \\ v_{2}=0 & \text { on }(T / 2, T) \times \partial \Omega \\ v_{2}(T / 2, .)=v_{1}(T / 2, .) & \text { in } \Omega\end{cases}
$$


satisfies

$$
v_{2}(T, .)=0 .
$$

From (23) and a Sobolev embedding (see for instance [30, Theorem 1.4.1] or [22, Lemma 3.3, page 80]), we set

$$
u_{2}:=H^{\frac{1}{2 k+1}} \in\left(\bigcap_{p \in[2,+\infty)} X_{(T / 2, T), p}\right) \subset L^{\infty}((T / 2, T) \times \Omega) .
$$

From (25) and (29), we have

$$
u_{2}(T / 2, .)=u_{2}(T, .)=0 .
$$

Then, we set, from (29)

$$
h_{2}:=\partial_{t} u_{2}-\Delta u_{2} \in \bigcap_{p \in[2,+\infty)} L^{p}((T / 2, T) \times \Omega),
$$

which is supported in $(T / 2, T) \times \omega)$ by $(26)$. Moreover, from (24) and (16), we get

$$
\forall p \in[2,+\infty),\left\|h_{2}\right\|_{L^{p}((T / 2, T) \times \Omega)} \leq C_{p}\left(\left\|u_{0}\right\|_{L^{\infty}(\Omega)}+\left\|v_{0}\right\|_{L^{\infty}(\Omega)}^{1 /(2 k+1)}\right) .
$$

By using (26), (27), (28), (29), (30), (31) and (32), we check that $\left(\left(u_{2}, v_{2}\right), h_{2}\right)$ satisfies Proposition 3.4.

3.3. Strategy of control in the whole interval $(0, T)$. We gather Proposition 3.1 and Proposition 3.4 to find a control which steers $(u, v)$ to $(0,0)$ in time $T$.

Proposition 3.10. There exists a control $h \in \bigcap_{p \in[2,+\infty)} L^{p}\left(Q_{T}\right)$ satisfying

$$
\forall p \in[2,+\infty),\|h\|_{L^{p}((0, T) \times \Omega)} \leq C_{p}\left(\left\|u_{0}\right\|_{L^{\infty}(\Omega)}+\left\|v_{0}\right\|_{L^{\infty}(\Omega)}^{1 /(2 k+1)}\right),
$$

such that the solution $(u, v) \in L^{\infty}\left(Q_{T}\right)$ of (Odd) satisfies $(u, v)(T,)=.(0,0)$.

Proof. Let $\left(\left(u_{1}, v_{1}\right), h_{1}\right) \in L^{\infty}((0, T / 2) \times \Omega)^{3}$ as in Proposition 3.1.

Let $\left(\left(u_{2}, v_{2}\right), h_{2}\right) \in L^{\infty}((T / 2, T) \times \Omega)^{2} \times \bigcap_{p \in[2,+\infty)} L^{p}((T / 2, T) \times \Omega)$ as in Proposition 3.4 .

We define $((u, v), h) \in\left(W_{T} \cap L^{\infty}\left(Q_{T}\right)\right)^{2} \times \bigcap_{p \in[2,+\infty)} L^{p}\left(Q_{T}\right)$ by

$$
\begin{array}{llll}
u=u_{1} & \text { in }[0, T / 2] \times \Omega, & u=u_{2} & \text { in }[T / 2, T] \times \Omega, \\
v=v_{1} & \text { in }[0, T / 2] \times \Omega, & v=v_{2} & \text { in }[T / 2, T] \times \Omega, \\
h=h_{1} & \text { in }(0, T / 2) \times \Omega, & h=h_{2} & \text { in }(T / 2, T) \times \Omega .
\end{array}
$$

We deduce from Proposition 3.1 and Proposition 3.4 that $(u, v) \in\left(W_{T} \cap L^{\infty}\left(Q_{T}\right)\right)^{2}$ is the solution of $(\mathrm{Odd})$ associated to the control $h$ and $(u, v)(T,)=.(0,0)$. Moreover, from the bounds (15) and (22), we get the bound (33).

Proposition 3.10 proves our first main result: Theorem 2.5.

\section{A control for the heat EQuation Which is AN odD power}

The goal of this section is to prove Proposition 3.7. We assume in the following that $\tau=T$. First, we prove a new Carleman estimate in $L^{2 k+2}$ for the heat equation. This type of inequality comes from the usual Carleman inequality in $L^{2}$ and parabolic regularity. Then, we get the existence of a control for the heat equation such that $h^{\frac{1}{2 k+1}}$ is regular by considering a penalized problem in $L^{\frac{2 k+2}{2 k+1}}$, which is a generalization of the usual Penalized Hilbert Uniqueness Method.

4.1. A Carleman inequality in $L^{2 k+2}$. 
4.1.1. Maximal regularity and Sobolev embeddings. We have the following parabolic regularity result and Sobolev embedding lemma.

Proposition 4.1. [14, Theorem 2.1]

Let $1<p<+\infty, g \in L^{p}\left(Q_{T}\right), y_{0} \in C_{0}^{\infty}(\Omega)$. The following Cauchy problem admits a unique solution $y \in X_{T, p}$ (see Definition 3.6)

$$
\begin{cases}\partial_{t} y-\Delta y=g & \text { in }(0, T) \times \Omega, \\ y=0 & \text { on }(0, T) \times \partial \Omega, \\ y(0, .)=y_{0} & \text { in } \Omega .\end{cases}
$$

Moreover, if $y_{0}=0$, there exists $C>0$ independent of $g$ such that

$$
\|y\|_{X_{T, p}} \leq C\|g\|_{L^{p}\left(Q_{T}\right)} .
$$

Lemma 4.2. [22, Lemma 3.3, page 80]

Let $p \in[1,+\infty)$, we have

$$
X_{T, p} \hookrightarrow\left\{\begin{array}{cl}
L^{\frac{(N+2) p}{N+2-p}}\left(0, T ; W_{0}^{1, \frac{(N+2) p}{N+2-p}}(\Omega)\right) & \text { if } p<N+2, \\
L^{2 p}\left(0, T ; W_{0}^{1,2 p}(\Omega)\right) & \text { if } p=N+2, \\
L^{\infty}\left(0, T ; W_{0}^{1, \infty}(\Omega)\right) & \text { if } p>N+2 .
\end{array}\right.
$$

4.1.2. Carleman estimates. We define

$$
\forall t \in(0, T), \eta(t):=\frac{1}{t(T-t)} .
$$

Let $\omega_{1}$ be a nonempty open subset such that

$$
\omega_{1} \subset \subset \omega .
$$

Let us recall the usual Carleman estimate in $L^{2}$ (see [11, Lemma 8] or [18] for a general introduction to Carleman estimates).

Proposition 4.3. [11, Lemma 8]

There exist $C>0$ and a function $\rho \in C^{2}(\bar{\Omega} ;(0,+\infty))$ such that for every $\varphi_{T} \in C_{0}^{\infty}(\Omega)$ and for every $s \geq C$, the solution $\varphi \in X_{T, 2}$ of

$$
\begin{cases}-\partial_{t} \varphi-\Delta \varphi=0 & (0, T) \times \Omega \\ \varphi=0 & (0, T) \times \partial \Omega \\ \varphi(T, .)=\varphi_{T} & \Omega\end{cases}
$$

satisfies

$$
\begin{aligned}
& \iint_{(0, T) \times \Omega} e^{-s \rho(x) \eta(t)}\left((s \eta)^{3}|\varphi|^{2}+(s \eta)|\nabla \varphi|^{2}\right) d x d t \\
& \leq C \iint_{(0, T) \times \omega_{1}} e^{-s \rho(x) \eta(t)}(s \eta)^{3}|\varphi|^{2} d x d t .
\end{aligned}
$$

From now on, $\rho$ is as in Proposition 4.3. We will deduce from the above $L^{2}$ Carleman estimate the following $L^{2 k+2}$-Carleman estimate.

Theorem 4.4. There exist $C>0$ and $m \in(0,+\infty)$ such that for every $\varphi_{T} \in C_{0}^{\infty}(\Omega)$ and for every $s \geq C$, the solution $\varphi \in X_{T, 2 k+2}$ of (36) satisfies

$$
\begin{aligned}
& \iint_{(0, T) \times \Omega} e^{-(k+1) s \rho(x) \eta(t)}\left((s \eta)^{-(k+1) m}|\varphi|^{2 k+2}+(s \eta)^{-(k+1)(m+2)}|\nabla \varphi|^{2 k+2}\right) d x d t \\
& \leq C \iint_{(0, T) \times \omega_{1}} e^{-(k+1) s \rho(x) \eta(t)}(s \eta)^{3(k+1)}|\varphi|^{2 k+2} d x d t .
\end{aligned}
$$


Proof. Let $\varphi_{T} \in C_{0}^{\infty}(\Omega), \varphi \in X_{T, 2 k+2}$ be the solution of (36) and $s \geq C$ where $C$ is as in Proposition 4.3.

By a standard parabolic regularity argument, one may deduce from the $L^{2}$-Carleman estimate (37) another Carleman type inequality in $L^{p_{0}}$ with $p_{0}=2(N+2) / N$. If $p_{0}>2 k+2$, this estimate implies (38). Otherwise, we iterate this strategy.

Step 1: We introduce the sequence $\left(\psi_{n}\right)_{n \geq-1}$,

$$
\psi_{-1}:=e^{-s \rho \eta / 2}(s \eta)^{3 / 2} \varphi, \quad \forall n \geq 0, \psi_{n}:=(s \eta)^{-2} \psi_{n-1}:=e^{-s \rho \eta / 2}(s \eta)^{3 / 2-2(n+1)} \varphi .
$$

Then, we also define an increasing sequence $\left(p_{n}\right)_{n \geq-1}$ by

$$
p_{-1}:=2, \quad \forall n \geq 0, p_{n}:=\left\{\begin{array}{cl}
\frac{(N+2) p_{n-1}}{N+2-p_{n-1}} & \text { if } p_{n-1}<N+2 \\
2 p_{n-1} & \text { if } p_{n-1}=N+2 \\
+\infty & \text { if } p_{n-1}>N+2
\end{array}\right.
$$

Clearly, there exists a unique integer $n_{0}$ such that

$$
p_{n_{0}}>2 k+2 \geq p_{n_{0}-1} .
$$

We will need this technical lemma.

Lemma 4.5. For every integer $n \in \mathbb{N}$,

$$
\begin{cases}-\partial_{t} \psi_{n}-\Delta \psi_{n}=g_{n} & \text { in }(0, T) \times \Omega, \\ \psi_{n}=0 & \text { on }(0, T) \times \partial \Omega, \\ \psi_{n}(T, .)=0 & \text { in } \Omega,\end{cases}
$$

with

$$
g_{n}(t, x)=a_{n}(t, x) \psi_{n-1}+(s \eta)^{-1} \nabla \psi_{n-1} \cdot \nabla \rho, \quad \text { where }\left\|a_{n}\right\|_{L^{\infty}\left(Q_{T}\right)} \leq C_{n} .
$$

Proof. We prove Lemma 4.5 by induction on $n \in \mathbb{N}$.

We introduce the notation

$$
\forall(t, x) \in(0, T) \times \Omega, \Theta(t, x):=e^{-s \rho(x) \eta(t) / 2}(s \eta(t))^{3 / 2} .
$$

Initialization: For $n=0$, by using (39), (44) and (36), we have

$-\partial_{t} \psi_{0}-\Delta \psi_{0}$

$=-\partial_{t}\left((s \eta)^{-2} \psi_{-1}\right)-\Delta\left((s \eta)^{-2} \psi_{-1}\right)$

$=-\partial_{t}\left((s \eta)^{-2}\right) \psi_{-1}+(s \eta)^{-2}\left(-\partial_{t} \psi_{-1}-\Delta \psi_{-1}\right)$

$=-\partial_{t}\left((s \eta)^{-2}\right) \psi_{-1}+(s \eta)^{-2}\left(-\left(\partial_{t} \Theta\right) \varphi+\Theta\left(-\partial_{t} \varphi-\Delta \varphi\right)-2 \nabla \Theta . \nabla \varphi-(\Delta \Theta) \varphi\right)$,

$$
-\partial_{t} \psi_{0}-\Delta \psi_{0}=-\partial_{t}\left((s \eta)^{-2}\right) \psi_{-1}+(s \eta)^{-2}\left(-\left(\partial_{t} \Theta\right) \varphi-2 \nabla \Theta \cdot \nabla \varphi-(\Delta \Theta) \varphi\right)
$$

Straightforward computations lead to

$$
\partial_{t} \Theta=e^{-s \rho \eta / 2}\left(-\frac{1}{2}\left(s \eta^{\prime}\right)(s \eta)^{3 / 2} \rho+\frac{3}{2}\left(s \eta^{\prime}\right)(s \eta)^{1 / 2}\right),
$$

$$
\nabla \Theta=-\frac{1}{2} e^{-s \rho \eta / 2}(s \eta)^{5 / 2} \nabla \rho, \quad \Delta \Theta=e^{-s \rho \eta / 2}\left(\frac{(s \eta)^{7 / 2}}{4}|\nabla \rho|^{2}-\frac{(s \eta)^{5 / 2}}{2} \Delta \rho\right)
$$


By using (45), (46), (47), we get

$$
\begin{aligned}
- & \partial_{t} \psi_{0}-\Delta \psi_{0} \\
= & -\partial_{t}\left((s \eta)^{-2}\right) \psi_{-1} \\
& +\left(e^{-s \rho \eta / 2}\left(\frac{1}{2}\left(s \eta^{\prime}\right)(s \eta)^{-1 / 2} \rho-\frac{3}{2}\left(s \eta^{\prime}\right)(s \eta)^{-3 / 2}-\frac{(s \eta)^{3 / 2}}{4}|\nabla \rho|^{2}+\frac{(s \eta)^{1 / 2}}{2} \Delta \rho\right)\right) \varphi \\
& +e^{-s \rho \eta / 2}(s \eta)^{1 / 2} \nabla \rho . \nabla \varphi .
\end{aligned}
$$

Moreover, by using (39) and (47), we have

$$
\begin{gathered}
\psi_{-1}=e^{-s \rho \eta / 2}(s \eta)^{3 / 2} \varphi \Leftrightarrow \varphi=e^{s \rho \eta / 2}(s \eta)^{-3 / 2} \psi_{-1}, \\
(s \eta)^{-1} \nabla \psi_{-1} \cdot \nabla \rho=(s \eta)^{-1}((\nabla \Theta . \nabla \rho) \varphi+(\nabla \varphi \cdot \nabla \rho) \Theta) \\
=e^{-s \rho \eta / 2}\left(-\frac{(s \eta)^{3 / 2}}{2}|\nabla \rho|^{2} \varphi+(s \eta)^{1 / 2} \nabla \rho . \nabla \varphi\right) .
\end{gathered}
$$

We gather (48), (49) and (50) to get (42) and (43) for $n=0$ (remark that $\eta^{\prime} \leq$ $\left.C\left(\eta^{2}+\eta^{3}\right)\right)$ with

$$
a_{0}:=-\partial_{t}\left((s \eta)^{-2}\right)+\eta^{\prime}\left(\frac{s^{-1} \eta^{-2}}{2} \rho-\frac{3}{2} s^{-2} \eta^{-3}\right)+\frac{1}{4}|\nabla \rho|^{2}+\frac{1}{2}(s \eta)^{-1} \Delta \rho \in L^{\infty}\left(Q_{T}\right),
$$

Heredity: Let $n \geq 1$. We assume that (42) and (43) hold true for $n-1$. Then, by using (39), we have

$$
\begin{aligned}
-\partial_{t} \psi_{n}-\Delta \psi_{n} & =-\partial_{t}\left((s \eta)^{-2} \psi_{n-1}\right)-\Delta\left((s \eta)^{-2} \psi_{n-1}\right) \\
& =-\partial_{t}\left((s \eta)^{-2}\right) \psi_{n-1}+(s \eta)^{-2}\left(-\partial_{t} \psi_{n-1}-\Delta \psi_{n-1}\right) \\
& =-\partial_{t}\left((s \eta)^{-2}\right) \psi_{n-1}+(s \eta)^{-2}\left(a_{n-1} \psi_{n-2}+(s \eta)^{-1} \nabla \psi_{n-2} . \nabla \rho\right) \\
& =-\partial_{t}\left((s \eta)^{-2}\right) \psi_{n-1}+a_{n-1} \psi_{n-1}+(s \eta)^{-1} \nabla \psi_{n-1} . \nabla \rho .
\end{aligned}
$$

Therefore, (42) and (43) hold true for $n$ with

$$
a_{n}(t, x):=-\partial_{t}\left((s \eta)^{-2}\right)+a_{n-1}(t, x) \in L^{\infty}\left(Q_{T}\right) .
$$

This ends the proof of Lemma 4.5.

Step 2: We show by induction that

$\forall n \in\left\{0, \ldots, n_{0}\right\}, \psi_{n} \in X_{T, p_{n-1}},\left\|\psi_{n}\right\|_{X_{T, p_{n-1}}}^{p_{n-1}} \leq C_{n}\left\|e^{-s \rho \eta / 2}(s \eta)^{3 / 2} \varphi\right\|_{L^{p_{n-1}\left((0, T) \times \omega_{1}\right)}}^{p_{n-1}}$.

First, we treat the case $n=0$. By using (43) for $n=0$, (49) and (50), we remark that

$$
\begin{aligned}
g_{0} & =a_{0} \psi_{-1}+(s \eta)^{-1} \nabla \psi_{-1} \cdot \nabla \rho \\
& =a_{0} e^{-s \rho \eta / 2}(s \eta)^{3 / 2} \varphi+e^{-s \rho \eta / 2}\left(-\frac{(s \eta)^{3 / 2}}{2}|\nabla \rho|^{2} \varphi+(s \eta)^{1 / 2} \nabla \rho . \nabla \varphi\right) .
\end{aligned}
$$

Then, from (54), we get that $g_{0} \in L^{2}\left(Q_{T}\right)$ and

$$
\left\|g_{0}\right\|_{L^{2}\left(Q_{T}\right)}^{2} \leq C \iint_{(0, T) \times \Omega} e^{-s \rho(x) \eta(t)}\left((s \eta)^{3}|\varphi|^{2}+(s \eta)|\nabla \varphi|^{2}\right) d x d t .
$$


Consequently, by (42) (for $n=0$ ), (55) and a parabolic regularity estimate (see Proposition 4.1 with $p=2$ ), we find that

(56) $\psi_{0} \in X_{T, 2}$ and $\left\|\psi_{0}\right\|_{X_{T, 2}}^{2} \leq C \iint_{(0, T) \times \Omega} e^{-s \rho(x) \eta(t)}\left((s \eta)^{3}|\varphi|^{2}+(s \eta)|\nabla \varphi|^{2}\right) d x d t$.

Gathering the Carleman estimate in $L^{2}$ i.e. (37) and (56), we have

$$
\left\|\psi_{0}\right\|_{X_{T, 2}}^{2} \leq C \iint_{(0, T) \times \omega_{1}} e^{-s \rho(x) \eta(t)}(s \eta)^{3}|\varphi|^{2} d x d t .
$$

This concludes the proof of (53) for $n=0$.

Now, we assume that (53) holds true for an integer $n \in\left\{0, \ldots, n_{0}-1\right\}$. By (40), a Sobolev embedding (see Lemma 4.2) applied to the left hand side of (53), the embedding $L^{p_{n}}\left((0, T) \times \omega_{1}\right) \hookrightarrow L^{p_{n-1}}\left((0, T) \times \omega_{1}\right)$, applied to right hand side of $(53)$, we obtain

$$
\begin{gathered}
\psi_{n} \in L^{p_{n}}\left(0, T ; W^{1, p_{n}}(\Omega)\right) \\
\left\|\psi_{n}\right\|_{L^{p_{n}}\left(0, T ; W^{\left.1, p_{n}(\Omega)\right)}\right.} \leq C_{n}\left\|e^{-s \rho \eta / 2}(s \eta)^{3 / 2} \varphi\right\|_{L^{p_{n}}\left((0, T) \times \omega_{1}\right)} .
\end{gathered}
$$

By using the parabolic equation satisfied by $\psi_{n+1}$ i.e. (42), (43) for $(n+1),(58)$ and a parabolic regularity estimate (see Proposition 4.1 with $p=p_{n}$ ), we get

$$
\psi_{n+1} \in X_{T, p_{n}} \quad \text { and } \quad\left\|\psi_{n+1}\right\|_{X_{T, p_{n}}}^{p_{n}} \leq C_{n+1}\left\|e^{-s \rho \eta / 2}(s \eta)^{3 / 2} \varphi\right\|_{L^{p_{n}}\left((0, T) \times \omega_{1}\right)}^{p_{n}} .
$$

This ends the proof of (53).

Step 3: We apply (53) with $n=n_{0}$ and we use a Sobolev embedding (see Lemma 4.2) and (40) to get

$$
\begin{gathered}
\psi_{n_{0}} \in L^{p_{n_{0}}}\left(0, T ; W^{1, p_{n_{0}}}(\Omega)\right) \\
\left\|\psi_{n_{0}}\right\|_{L^{p_{n_{0}}\left(0, T ; W^{1, p_{0}}(\Omega)\right)}} \leq C\left\|e^{-s \rho \eta / 2}(s \eta)^{3 / 2} \varphi\right\|_{L^{p_{n_{0}-1}\left((0, T) \times \omega_{1}\right)}} .
\end{gathered}
$$

Recalling the definition of $n_{0}$ in (41), and by using (59), together with the embedding

$$
L^{p_{n}}\left(Q_{T}\right) \hookrightarrow L^{2 k+2}\left(Q_{T}\right),
$$

applied to the left hand side of (59) and the embedding

$$
L^{2 k+2}\left((0, T) \times \omega_{1}\right) \hookrightarrow L^{p_{n_{0}-1}}\left((0, T) \times \omega_{1}\right),
$$

applied to the right hand side of (59), we get

$$
\left\|\psi_{n_{0}}\right\|_{L^{2 k+2}\left(Q_{T}\right)}^{p_{n_{0}-1}}+\left\|\nabla \psi_{n_{0}}\right\|_{L^{2 k+2}\left(Q_{T}\right)}^{p_{n_{0}-1}} \leq C\left\|e^{-s \rho \eta / 2}(s \eta)^{3 / 2} \varphi\right\|_{L^{2 k+2}\left((0, T) \times \omega_{1}\right)}^{p_{n_{0}-1}} .
$$

Then, from the definition (39) of $\psi_{n_{0}}$, we get

$$
\begin{gathered}
\psi_{n_{0}}=e^{-s \rho \eta / 2}(s \eta)^{-1 / 2-2 n_{0}} \varphi \\
\nabla \psi_{n_{0}}=-\frac{1}{2} e^{-s \rho \eta / 2}(s \eta)^{1 / 2-2 n_{0}} \varphi \nabla \rho+e^{-s \rho \eta / 2}(s \eta)^{-1 / 2-2 n_{0}} \nabla \varphi .
\end{gathered}
$$

Consequently, we deduce from (61) and (62) that

$$
\begin{aligned}
& \left\|e^{-s \rho \eta / 2}(s \eta)^{-1 / 2-2 n_{0}} \varphi\right\|_{L^{2 k+2}\left(Q_{T}\right)}^{p_{n_{0}-1}}+\left\|e^{-s \rho \eta / 2}(s \eta)^{-3 / 2-2 n_{0}} \nabla \varphi\right\|_{L^{2 k+2}\left(Q_{T}\right)}^{p_{n_{0}-1}} \\
& \leq C\left(\left\|\psi_{n_{0}}\right\|_{L^{2 k+2}\left(Q_{T}\right)}^{p_{n_{0}-1}}+\left\|(s \eta)^{-1} \nabla \psi_{n_{0}}\right\|_{L^{2 k+2}\left(Q_{T}\right)}^{p_{n_{0}-1}}\right) \\
& \leq C\left(\left\|\psi_{n_{0}}\right\|_{L^{2 k+2}\left(Q_{T}\right)}^{p_{n_{0}-1}}+\left\|\nabla \psi_{n_{0}}\right\|_{L^{2 k+2}\left(Q_{T}\right)}^{p_{n_{0}-1}}\right) .
\end{aligned}
$$

By using (60) and (63), we get (38) with $m=4 n_{0}+1$. 
We consider $\chi \in C^{\infty}(\bar{\Omega} ;[0,+\infty))$ such that

$$
\operatorname{supp}(\chi) \subset \subset \omega, \quad \chi=1 \text { in } \omega_{1}, \quad \chi^{\frac{1}{2 k+1}} \in C^{\infty}(\bar{\Omega} ;[0,+\infty)) .
$$

We deduce from Theorem 4.4 the following result.

Corollary 4.6. There exist $C>0$ and $m \in(0,+\infty)$ such that for every $\varphi_{T} \in L^{2 k+2}(\Omega)$ and for every $s \geq C$, the solution $\varphi \in L^{2 k+2}\left(Q_{T}\right)$ of (36) satisfies

$$
\begin{aligned}
& \iint_{(0, T) \times \Omega} e^{-(k+1) s \rho(x) \eta(t)}\left((s \eta)^{-(k+1) m}|\varphi|^{2 k+2}+(s \eta)^{-(k+1)(m+2)}|\nabla \varphi|^{2 k+2}\right) d x d t \\
& \leq C \iint_{(0, T) \times \omega} \chi^{2 k+2} e^{-(k+1) s \rho(x) \eta(t)}(s \eta)^{3(k+1)}|\varphi|^{2 k+2} d x d t
\end{aligned}
$$

and

(66) $\|\varphi(0, .)\|_{L^{2 k+2}(\Omega)}^{2 k+2} \leq C_{s} \iint_{(0, T) \times \omega} \chi^{2 k+2} e^{-(k+1) s \rho(x) \eta(t)}(s \eta)^{3(k+1)}|\varphi|^{2 k+2} d x d t$.

Remark 4.7. We insist on the fact that the constant $C$ of the observability inequality (66) depends on the parameter $s$. It is not the case of (65).

Proof. Step 1: We assume that $\varphi_{T} \in C_{0}^{\infty}(\Omega)$. We denote by $\varphi \in X_{T, 2 k+2}$, the solution of (36). Then, by Theorem 4.4 and (64), for every $s \geq C$, (65) holds and in particular,

$$
\begin{aligned}
& \iint_{(0, T) \times \Omega} e^{-(k+1) s \rho \eta}(s \eta)^{-(k+1) m}|\varphi|^{2 k+2} \\
& \leq C \iint_{(0, T) \times \omega} \chi^{2 k+2} e^{-(k+1) s \rho \eta}(s \eta)^{3(k+1)}|\varphi|^{2 k+2}
\end{aligned}
$$

We fix $s$ sufficiently large such that (67) holds.

By using

$$
\min _{[T / 4,3 T / 4] \times \bar{\Omega}} e^{-(k+1) s \rho(x) \eta(t)}(s \eta)^{-(k+1) m}>0,
$$

together with (67), we get

$$
\int_{T / 4}^{3 T / 4} \int_{\Omega}|\varphi|^{2 k+2} d x d t \leq C_{s} \iint_{(0, T) \times \omega} \chi^{2 k+2} e^{-(k+1) s \rho(x) \eta(t)}(s \eta)^{3(k+1)}|\varphi|^{2 k+2} d x d t .
$$

From the dissipation of the $L^{2 k+2}$-norm for (36) (obtained by multiplying the equation (36) by $|\varphi|^{p-2} \varphi$ and integrating over $\left.\Omega\right):\|\varphi(0, .)\|_{L^{2 k+2}(\Omega)} \leq\|\varphi(t, .)\|_{L^{2 k+2}(\Omega)}$ for $t \in(T / 4,3 T / 4)$, by integrating in time, we get

$$
\|\varphi(0, .)\|_{L^{2 k+2}(\Omega)}^{2 k+2} \leq C \int_{T / 4}^{3 T / 4} \int_{\Omega}|\varphi|^{2 k+2} d x d t .
$$

Gathering (69) and (70), we get (66).

Step 2: The general case comes from a density argument by using in particular Definition-Proposition 2.3: (7) for $p=2 k+2$. The complete proof is reported to Appendix A.2. 
4.2. A new penalized duality method in $L^{(2 k+2) /(2 k+1)}$, the Reflexive Uniqueness Method. From now on, $\chi$ is a function which belongs to $C^{\infty}(\bar{\Omega} ;[0,+\infty))$ satisfying (64) and $m, s$ are fixed by Corollary 4.6.

We introduce the notations

$$
q:=\frac{2 k+2}{2 k+1}
$$

$$
L_{w g h t}^{q}((0, T) \times \omega):=\left\{h \in L^{q}((0, T) \times \omega) ; e^{s p \eta / 2}(s \eta)^{-3 / 2} h \in L^{q}((0, T) \times \omega)\right\} .
$$

The goal of this section is to get a null-controllability result for the heat equation thanks to the observability inequalities of Corollary 4.6.

Proposition 4.8. For every $\zeta_{0} \in L^{q}(\Omega)$, there exists a control $h \in L_{w g h t}^{q}((0, T) \times \omega)$ such that the solution $\zeta$ of

$$
\begin{cases}\partial_{t} \zeta-\Delta \zeta=h \chi & \text { in }(0, T) \times \Omega \\ \zeta=0 & \text { on }(0, T) \times \partial \Omega \\ \zeta(0, .)=\zeta_{0} & \text { in } \Omega\end{cases}
$$

satisfies $\zeta(T,)=$.0 and

$$
\left\|e^{s \rho(x) \eta(t) / 2}(s \eta)^{-3 / 2} h\right\|_{L^{q}((0, T) \times \omega)} \leq C\left\|\zeta_{0}\right\|_{L^{q}(\Omega)} .
$$

Proof. Let $\zeta_{0} \in C_{0}^{\infty}(\Omega)$. The general case comes from a density argument.

We first state two easy facts.

Fact 4.9. The antiderivative of the continuous mapping $x \in \mathbb{R} \mapsto x^{\frac{1}{2 k+1}}$ (see Definition 3.5$)$ is the strictly convex function

$$
x \in \mathbb{R} \mapsto \frac{1}{q}|x|^{q}:=\left\{\begin{array}{cl}
\frac{1}{q} \exp (q \log (|x|)) & \text { if } x \neq 0, \\
0 & \text { if } x=0 .
\end{array}\right.
$$

Fact 4.10. The real numbers $2 k+2, q$ belong to $(1,+\infty)$ and are conjugate:

$$
\frac{1}{2 k+2}+\frac{1}{q}=1 \text {. }
$$

Let $\varepsilon \in(0,1)$. We consider the minimization problem

$$
\inf _{h \in L_{\text {wght }}^{q}((0, T) \times \omega)} J(h),
$$

where $J$ is defined as follows: for every $h \in L_{w g h t}^{q}((0, T) \times \omega)$,

$$
J(h):=\frac{1}{q} \iint_{(0, T) \times \omega} e^{(q / 2) s \rho(x) \eta(t)}(s \eta)^{-3 q / 2}|h|^{q} d x d t+\frac{1}{q \varepsilon}\|\zeta(T, .)\|_{\left.L^{q} \Omega\right)}^{q},
$$

where $\zeta \in X_{T, q}$ is the solution of (72) (see Proposition 4.1).

The mapping $J$ is a coercive, strictly convex (see Fact 4.9), $C^{1}$ function on the reflexive space $L_{w g h t}^{q}((0, T) \times \omega)$. Then, $J$ has a unique minimum $h^{\varepsilon}$. We denote by $\zeta^{\varepsilon} \in X_{T, q}$ the solution of (72) associated to the control $h^{\varepsilon}$. The Euler-Lagrange equation gives

$$
\begin{aligned}
\forall h \in L_{w g h t}^{q}((0, T) \times \omega), & \iint_{(0, T) \times \omega} e^{(q / 2) s \rho(x) \eta(t)}(s \eta)^{-3 q / 2}\left(h^{\varepsilon}\right)^{1 /(2 k+1)} h d x d t \\
& +\frac{1}{\varepsilon} \int_{\Omega}\left(\zeta^{\varepsilon}(T, x)\right)^{1 /(2 k+1)} \zeta(T, x) d x=0
\end{aligned}
$$

where $\zeta \in X_{T, q}$ is the solution of (72) (associated to the control $h$ ) with $\zeta_{0}=0$.

We introduce $\varphi^{\varepsilon} \in L^{2 k+2}\left(Q_{T}\right)$ the solution of the adjoint problem

$$
\begin{cases}-\partial_{t} \varphi^{\varepsilon}-\Delta \varphi^{\varepsilon}=0 & \text { in }(0, T) \times \Omega \\ \varphi^{\varepsilon}=0 & \text { on }(0, T) \times \partial \Omega \\ \varphi^{\varepsilon}(T, .)=-\frac{1}{\varepsilon}\left(\zeta^{\varepsilon}(T, .)\right)^{1 /(2 k+1)}=: \varphi_{T}^{\varepsilon} & \text { in } \Omega\end{cases}
$$


By a duality argument between $\zeta$, the solution of (72) with $\zeta_{0}=0$ and $\varphi^{\epsilon}$, we have

$$
\forall h \in L_{w g h t}^{q}((0, T) \times \omega), \quad \int_{\Omega} \varphi^{\varepsilon}(T, x) \zeta(T, x) d x=\iint_{(0, T) \times \omega} \varphi^{\varepsilon} h \chi d x d t .
$$

Indeed, first, one can prove the result for $\varphi_{T}^{\varepsilon} \in C_{0}^{\infty}(\Omega)$ because in this case $\varphi^{\varepsilon} \in$ $X_{T, 2 k+2}$ and $\zeta \in X_{T, q}$. This justifies the calculations for the duality argument. Then, the fact that ${\overline{C_{0}^{\infty}(\Omega)}}^{L^{2 k+2}(\Omega)}=L^{2 k+2}(\Omega)$ leads to $(78)$.

From (77) (definition of $\varphi_{T}^{\varepsilon}$ ) and (78), we have

$$
\forall h \in L_{w g h t}^{q}((0, T) \times \omega),-\frac{1}{\varepsilon} \int_{\Omega}\left(\zeta^{\varepsilon}(T, x)\right)^{1 /(2 k+1)} \zeta(T, x) d x=\iint_{(0, T) \times \omega} \varphi^{\varepsilon} h \chi d x d t .
$$

Then, by using (76) and (79), we obtain

$$
\left(h^{\varepsilon}\right)^{1 /(2 k+1)}=e^{-(q / 2) s \rho(x) \eta(t)}(s \eta)^{3 q / 2} \varphi^{\varepsilon} \chi .
$$

Moreover, from a duality argument between $\varphi^{\varepsilon}$ and $\zeta^{\varepsilon}$, together with (80), we have

$$
\begin{aligned}
& \int_{\Omega} \varphi^{\varepsilon}(T, x) \zeta^{\varepsilon}(T, x) d x \\
& =-\frac{1}{\varepsilon}\left\|\zeta^{\varepsilon}(T, .)\right\|_{L^{q}(\Omega)}^{q} \\
& =\iint_{(0, T) \times \omega} \varphi^{\varepsilon} h^{\varepsilon} \chi d x d t+\int_{\Omega} \varphi^{\varepsilon}(0, x) \zeta_{0}(x) d x \\
& =\iint_{(0, T) \times \omega} e^{-(k+1) s \rho \eta}(s \eta)^{3(k+1)}\left|\varphi^{\varepsilon}\right|^{2 k+2} \chi^{2 k+2}+\int_{\Omega} \varphi^{\varepsilon}(0, .) \zeta_{0}(.) .
\end{aligned}
$$

By Young's inequality, we have for every $\delta>0$,

$$
\left|\int_{\Omega} \varphi^{\varepsilon}(0, x) \zeta_{0}(x) d x\right| \leq \delta\left\|\varphi^{\varepsilon}(0, .)\right\|_{L^{2 k+2}(\Omega)}^{2 k+2}+C_{\delta}\|\zeta(0, .)\|_{L^{q}(\Omega)}^{q} .
$$

From (81), (82), the observability inequality (66) (applied to $\varphi^{\varepsilon}$ ), and by taking $\delta$ sufficiently small, we get

$$
\frac{1}{\varepsilon}\left\|\zeta^{\varepsilon}(T, .)\right\|_{L^{q}(\Omega)}^{q}+\iint_{(0, T) \times \omega} e^{-(k+1) s \rho \eta}(s \eta)^{3(k+1)}\left|\varphi^{\varepsilon}\right|^{2 k+2} \chi^{2 k+2} \leq C\left\|\zeta_{0}\right\|_{L^{q}(\Omega)}^{q} .
$$

Now, plugging (80) into (83), we obtain

$$
\frac{1}{\varepsilon}\left\|\zeta^{\varepsilon}(T, .)\right\|_{L^{q}(\Omega)}^{q}+\left\|e^{s \rho(x) \eta(t) / 2}(s \eta)^{-3 / 2} h^{\varepsilon}\right\|_{L^{q}((0, T) \times \omega)}^{q} \leq C\left\|\zeta_{0}\right\|_{L^{q}(\Omega)}^{q} .
$$

In particular, from (84), we have

$$
\zeta^{\varepsilon}(T, .) \underset{\varepsilon \rightarrow 0}{\rightarrow} 0 \text { in } L^{q}(\Omega)
$$

and

$$
\left\|h^{\varepsilon}\right\|_{L^{q}\left(Q_{T}\right)} \leq C
$$

We remark that

$$
\zeta^{\varepsilon}=\zeta_{1}^{\varepsilon}+\zeta_{2}^{\varepsilon}
$$

with $\zeta_{1}^{\varepsilon}, \zeta_{2}^{\varepsilon}$ satisfying

$$
\left\{\begin{array} { l l } 
{ \partial _ { t } \zeta _ { 1 } ^ { \varepsilon } - \Delta \zeta _ { 1 } ^ { \varepsilon } = 0 } & { \text { in } ( 0 , T ) \times \Omega , } \\
{ \zeta _ { 1 } ^ { \varepsilon } = 0 } & { \text { on } ( 0 , T ) \times \partial \Omega , } \\
{ \zeta _ { 1 } ^ { \varepsilon } = \zeta _ { 0 } } & { \text { in } \Omega , }
\end{array} \quad \left\{\begin{array}{ll}
\partial_{t} \zeta_{2}^{\varepsilon}-\Delta \zeta_{2}^{\varepsilon}=h^{\varepsilon} \chi & \text { in }(0, T) \times \Omega, \\
\zeta_{2}^{\varepsilon}=0 & \text { on }(0, T) \times \partial \Omega, \\
\zeta_{2}^{\varepsilon}=0 & \text { in } \Omega .
\end{array}\right.\right.
$$


Then, by using (87), (88), (86) and Proposition 4.1 with $p=q$, we have

$$
\left\|\zeta^{\varepsilon}\right\|_{X_{T, q}} \leq C
$$

So, from (89), up to a subsequence, we can suppose that there exists $\zeta \in X_{T, q}$ such that

$$
\zeta^{\varepsilon} \underset{\varepsilon \rightarrow 0}{\rightarrow} \zeta \text { in } X_{T, q},
$$

and from the embedding $X_{T, q} \hookrightarrow C\left([0, T] ; L^{q}(\Omega)\right.$ ) (see [16, Section 5.9.2, Theorem 2]),

$$
\zeta^{\varepsilon}(0, .) \underset{\varepsilon \rightarrow 0}{\rightarrow} \zeta(0, .) \text { in } L^{q}(\Omega), \zeta^{\varepsilon}(T, .) \underset{\varepsilon \rightarrow 0}{\rightarrow} \zeta(T, .) \text { in } L^{q}(\Omega) .
$$

Then, as we have $\zeta^{\varepsilon}(0,)=.\zeta_{0}$ and $(85)$, we deduce that

$$
\zeta(0, .)=\zeta_{0} \text { and } \zeta(T, .)=0 .
$$

Moreover, from (84), up to a subsequence, we can suppose that there exists $h \in$ $L_{w g h t}^{q}((0, T) \times \omega)$ such that

$$
h^{\varepsilon} \underset{\varepsilon \rightarrow 0}{\rightarrow} h \text { in } L_{w g h t}^{q}((0, T) \times \omega),
$$

and

$$
\text { (94) } \begin{aligned}
\left\|e^{s \rho(x) \eta(t) / 2}(s \eta)^{-3 / 2} h\right\|_{L^{q}((0, T) \times \omega)}^{q} & \leq \lim _{\varepsilon \rightarrow 0} \inf \left\|e^{s \rho(x) \eta(t) / 2}(s \eta)^{-3 / 2} h^{\varepsilon}\right\|_{L^{q}((0, T) \times \omega)}^{q} \\
& \leq C\left\|\zeta_{0}\right\|_{L^{q}(\Omega)}^{q} .
\end{aligned}
$$

Then, from (90), (93), and (91), we let $\varepsilon \rightarrow 0$ in the following equations

$$
\begin{cases}\partial_{t} \zeta^{\varepsilon}-\Delta \zeta^{\varepsilon}=h^{\varepsilon} \chi & \text { in }(0, T) \times \Omega, \\ \zeta^{\varepsilon}=0 & \text { on }(0, T) \times \partial \Omega, \\ \zeta^{\varepsilon}(0, .)=\zeta_{0} & \text { in } \Omega,\end{cases}
$$

and by using (92), we deduce

$$
\begin{cases}\partial_{t} \zeta-\Delta \zeta=h \chi & \text { in }(0, T) \times \Omega \\ \zeta=0 & \text { on }(0, T) \times \partial \Omega \\ (\zeta(0, .), \zeta(T, .))=\left(\zeta_{0}, 0\right) & \text { in } \Omega\end{cases}
$$

Therefore, (96) and (94) conclude the proof of Proposition 4.8.

4.3. A Bootstrap argument. The goal of this section is to prove Proposition 3.7. We keep the same notations as in the proof of Proposition 4.8. We want to improve the regularity of the sequence $\left(\left(h^{\varepsilon}\right)^{\frac{1}{2 k+1}}\right)_{\varepsilon>0}$. The key point is the equality (80). We deduce that the regularity of $\left(h^{\varepsilon}\right)^{\frac{1}{2 k+1}}$ depends on the regularity of $e^{-(q / 2) s \rho(x) \eta(t)}(s \eta)^{3 q / 2} \varphi^{\varepsilon}$. We use parabolic regularity estimates (see Proposition 4.1) and a bootstrap argument (similar to the proof of Theorem 4.4). The starting point is $(65)$.

Step 1: We introduce the sequence $\left(\psi_{n}^{\varepsilon}\right)_{n \geq-1}$,

$\psi_{-1}^{\varepsilon}:=e^{-s \rho \eta / 2}(s \eta)^{-m / 2} \varphi, \quad \forall n \geq 0, \psi_{n}:=(s \eta)^{-2} \psi_{n-1}^{\varepsilon}=e^{-s \rho \eta / 2}(s \eta)^{-m / 2-2(n+1)} \varphi^{\varepsilon}$, where $m$ is defined in Corollary 4.6. Then, we also define an increasing sequence $\left(p_{n}\right)_{n \geq-1}$ by

$$
p_{-1}:=2 k+2, \quad \forall n \geq 0, p_{n}:=\left\{\begin{array}{cl}
\frac{(N+2) p_{n-1}}{N+2-p_{n-1}} & \text { if } p_{n-1}<N+2, \\
2 p_{n-1} & \text { if } p_{n-1}=N+2, \\
+\infty & \text { if } p_{n-1}>N+2 .
\end{array}\right.
$$

We denote by $l$ the integer such that

$$
l:=\min \left\{n \in \mathbb{N} ; p_{n}=+\infty\right\} .
$$


By using (97) and (77), we show by induction that for every $n \in \mathbb{N}$,

$$
\begin{cases}-\partial_{t} \psi_{n}^{\varepsilon}-\Delta \psi_{n}^{\varepsilon}=g_{n}^{\varepsilon} & \text { in }(0, T) \times \Omega, \\ \psi_{n}^{\varepsilon}=0 & \text { on }(0, T) \times \partial \Omega, \\ \psi_{n}^{\varepsilon}(T, .)=0 & \text { in } \Omega,\end{cases}
$$

with

(101) $g_{n}(t, x)=a_{n}(t, x) \psi_{n-1}^{\varepsilon}+(s \eta)^{-1} \nabla \psi_{n-1}^{\varepsilon} . \nabla \rho, \quad$ where $\left\|a_{n}\right\|_{L^{\infty}\left(Q_{T}\right)} \leq C_{n}$.

Indeed, straightforward computations as in the proof of Lemma 4.5 lead to

$$
\begin{gathered}
a_{0}:=-\partial_{t}\left((s \eta)^{-2}\right)+\eta^{\prime}\left(\frac{s^{-1} \eta^{-2}}{2} \rho+\frac{m}{2} s^{-2} \eta^{-3}\right)+\frac{1}{4}|\nabla \rho|^{2}+\frac{1}{2}(s \eta)^{-1} \Delta \rho, \\
a_{n}:=-\partial_{t}\left((s \eta)^{-2}\right)+a_{n-1} .
\end{gathered}
$$

Step 2: From (65), (83), (97) and (71), we have

$$
\left\|\psi_{-1}\right\|_{L^{2 k+2}\left(Q_{T}\right)}+\left\|(s \eta)^{-1} \nabla \psi_{-1}\right\|_{L^{2 k+2}\left(Q_{T}\right)} \leq C\left\|\zeta_{0}\right\|_{L^{q}(\Omega)}^{q /(2 k+2)}=C\left\|\zeta_{0}\right\|_{L^{q}(\Omega)}^{1 /(2 k+1)} .
$$

Then, by using parabolic regularity estimate (see Proposition 4.1), (100), (101), (104) and an induction argument (as in the proof of Theorem 4.4), we have that

$$
\forall n \in\{0, \ldots, l\}, \psi_{n}^{\varepsilon} \in X_{T, p_{n-1}} \quad \text { and } \quad\left\|\psi_{n}^{\varepsilon}\right\|_{X_{T, p_{n-1}}} \leq C_{n}\left\|\zeta_{0}\right\|_{L^{q}(\Omega)}^{1 /(2 k+1)} .
$$

Step 3: We apply (105) with $n=l$ (see (99)) and we use Lemma 4.2 with $p=p_{l-1}$ to get

(106) $\psi_{l}^{\varepsilon} \in L^{\infty}\left(0, T ; W_{0}^{1, \infty}(\Omega)\right) \quad$ and $\quad\left\|\psi_{l}^{\varepsilon}\right\|_{L^{\infty}\left(0, T ; W_{0}^{1, \infty}(\Omega)\right)} \leq C\left\|\zeta_{0}\right\|_{L^{q}(\Omega)}^{1 /(2 k+1)}$.

From a parabolic regularity estimate (see Proposition 4.1) applied to the heat equation satisfied by $\psi_{l+1}^{\varepsilon}$ and (106), we obtain

(107) $\psi_{l+1}^{\varepsilon} \in \cap_{p \in[2,+\infty)} X_{T, p} \quad$ and $\quad \forall p \in[2,+\infty),\left\|\psi_{l+1}^{\varepsilon}\right\|_{X_{T, p}} \leq C_{p}\left\|\zeta_{0}\right\|_{L^{q}(\Omega)}^{1 /(2 k+1)}$.

From (80), (97) (see in particular that $q>1$ ), we have

$$
\forall p \in[2,+\infty),\left\|\left(h^{\varepsilon}\right)^{1 /(2 k+1)}\right\|_{X_{T, p}} \leq C_{p}\left\|\psi_{l+1}^{\varepsilon}\right\|_{X_{T, p}} .
$$

From (107) and (108), we have

$$
\left(h^{\varepsilon}\right)^{1 /(2 k+1)} \in \bigcap_{p \in[2,+\infty)} X_{T, p}, \forall p \in[2,+\infty),\left\|\left(h^{\varepsilon}\right)^{1 /(2 k+1)}\right\|_{X_{T, p}} \leq C_{p}\left\|\zeta_{0}\right\|_{L^{q}(\Omega)}^{1 /(2 k+1)} .
$$

Now, by (84) and (109), we have

$$
\forall p \in[2,+\infty), \frac{1}{\varepsilon^{1 /(2 k+2)}}\left\|\zeta^{\varepsilon}(T, .)\right\|_{L^{q}(\Omega)}^{1 /(2 k+1)}+\left\|\left(h^{\varepsilon}\right)^{1 /(2 k+1)}\right\|_{X_{T, p}} \leq C_{p}\left\|\zeta_{0}\right\|_{L^{q}(\Omega)}^{1 /(2 k+1)} .
$$

Step 4: From (110) and same arguments given as previously (see Section 4.2), together with a diagonal extraction process, up to a subsequence, we can assume that there exist $H \in \cap_{p \in[2,+\infty)} X_{T, p}$ and $\zeta \in X_{T, p}$ such that

$$
\begin{aligned}
& \left(h^{\varepsilon}\right)^{1 /(2 k+1)} \underset{\varepsilon \rightarrow 0}{\rightarrow} H \text { in } X_{T, p} \forall p \in[2,+\infty), \\
& \left(h^{\varepsilon}\right)^{1 /(2 k+1)} \underset{\varepsilon \rightarrow 0}{\rightarrow} H \text { in } L^{\infty}\left(Q_{T}\right), \quad\left(\Rightarrow h^{\varepsilon} \underset{\varepsilon \rightarrow 0}{\rightarrow} H^{2 k+1} \text { in } L^{\infty}\left(Q_{T}\right)\right), \\
& H(0, .)=0, \quad H(T, .)=0, \quad(\text { see }(80)), \\
& \zeta^{\varepsilon} \underset{\varepsilon \rightarrow 0}{\rightarrow} \zeta \text { in } X_{T, q}, \\
& \zeta(0, .)=\zeta_{0}, \quad \zeta(T, .)=0 .
\end{aligned}
$$

The strong $L^{\infty}$-convergence $(112)$ is a consequence of the weak $X_{T, p^{-}}$-convergence (111) for $p$ sufficiently large because $X_{T, p}$ is relatively compact in $L^{\infty}\left(Q_{T}\right)$ (see $[28$, 
Section 8, Corollary 4]: Aubin-Lions lemma).

By using (112), (114) and (115) and by letting $\varepsilon \rightarrow 0$ in the following equations

$$
\begin{cases}\partial_{t} \zeta^{\varepsilon}-\Delta \zeta^{\varepsilon}=h^{\varepsilon} \chi & \text { in }(0, T) \times \Omega, \\ \zeta^{\varepsilon}=0 & \text { on }(0, T) \times \partial \Omega, \\ \zeta^{\varepsilon}(0, .)=\zeta_{0} & \text { in } \Omega,\end{cases}
$$

we deduce

$$
\begin{cases}\partial_{t} \zeta-\Delta \zeta=H^{2 k+1} \chi & \text { in }(0, T) \times \Omega, \\ \zeta=0 & \text { on }(0, T) \times \partial \Omega, \\ (\zeta(0, .), \zeta(T, .))=\left(\zeta_{0}, 0\right) & \text { in } \Omega\end{cases}
$$

To sum up, for all $\zeta_{0} \in C_{0}^{\infty}(\Omega)$, we have found a control

$$
h:=H^{2 k+1} \chi \in L^{\infty}\left(Q_{T}\right),
$$

such that

$$
\begin{aligned}
& h^{1 /(2 k+1)}=H \chi^{1 /(2 k+1)} \in \bigcap_{[2,+\infty)} X_{T, p}, \quad(\text { see }(111)), \\
& \forall p \in[2,+\infty),\left\|h^{1 /(2 k+1)}\right\|_{X_{T, p}} \leq C_{p}\left\|\zeta_{0}\right\|_{L^{q}(\Omega)}^{1 /(2 k+1)}, \quad(\text { see }(110)), \\
& h(0, .)=h(T, .)=0, \quad(\text { see }(113)) .
\end{aligned}
$$

Moreover, from (117), the solution $\zeta \in L^{\infty}\left(Q_{T}\right)$ of

$$
\begin{cases}\partial_{t} \zeta-\Delta \zeta=h & \text { in }(0, T) \times \Omega, \\ \zeta=0 & \text { on }(0, T) \times \partial \Omega, \\ \zeta(0, .)=\zeta_{0} & \text { in } \Omega,\end{cases}
$$

satisfies

$$
\zeta(T, .)=0 .
$$

By (118), (119), (120), (121), (122) and (123), we deduce Proposition 3.7

for $\zeta_{0} \in C_{0}^{\infty}(\Omega)$. The general case comes from ${\overline{C_{0}^{\infty}(\Omega)}}^{L^{q}(\Omega)}=L^{q}(\Omega)$ and the bound (119).

Remark 4.11. In this paragraph, we gives the main details to get Remark 3.9 and consequently Remark 2.7. By (12), the function $\rho$, as in Proposition 4.3, can be chosen such that

$$
\rho \in C^{2, \alpha}(\bar{\Omega})
$$

see the proof of [9, Lemma 2.68].

Let us take $\beta$ such that $1+\alpha \leq \beta<2$. From Sobolev embedding (see [30, Corollary 1.4.1]) and (107), we have for $p$ sufficiently large,

$$
\psi_{l+1}^{\varepsilon} \in X_{T, p} \hookrightarrow C^{\beta / 2, \beta}\left(\overline{Q_{T}}\right) \text { and }\left\|\psi_{l+1}^{\varepsilon}\right\|_{C^{\beta / 2, \beta}\left(\overline{Q_{T}}\right)} \leq C\left\|\zeta_{0}\right\|_{L^{q}(\Omega)}^{1 /(2 k+1)} .
$$

From (124), (102) and (103), we have $a_{l+2} \in C^{\alpha / 2, \alpha}\left(\overline{Q_{T}}\right)$. Then, we deduce from (100), (101) for $n=l+2,(125)$, (12) and a parabolic regularity theorem in Hölder spaces (see [30, Theorem 8.3.7 and Theorem 7.2.24]) that $\psi_{l+2}^{\varepsilon} \in C^{1+\alpha / 2,2+\alpha}\left(\overline{Q_{T}}\right)$. Therefore, we have

$$
\frac{1}{\varepsilon^{1 /(2 k+2)}}\left\|\zeta^{\varepsilon}(T, .)\right\|_{L^{q}(\Omega)}^{1 /(2 k+1)}+\left\|\left(h^{\varepsilon}\right)^{1 /(2 k+1)}\right\|_{C^{1+\alpha / 2,2+\alpha}\left(\overline{Q_{T}}\right)} \leq C\left\|\zeta_{0}\right\|_{L^{q}(\Omega)}^{1 /(2 k+1)} .
$$

By (126), we conclude the proof of Remark 3.9 as in the Step 4 by using the compact embedding $C^{1+\alpha / 2,2+\alpha}\left(\overline{Q_{T}}\right) \hookrightarrow C^{1,2}\left(\overline{Q_{T}}\right)$ by Ascoli's theorem. 


\section{LOCAL NULL-CONTROLLABILITY OF GENERAL NONLINEAR SYSTEMS}

The proof of Theorem 2.8 relies on the return method and Proposition 1.7. Thus, we just need to construct an appropriate reference trajectory $((\bar{u}, \bar{v}), \bar{h})$. The goal of this section is to prove the existence of a nontrivial trajectory of (NL) associated to $f_{1}$ and $f_{2}$ defined in Theorem 2.8 (see in particular (13) and (14)). More precisely, we have the following result.

Proposition 5.1. Let $\omega_{0}$ be a nonempty open subset such that $\omega_{0} \subset \subset \omega$. There exist $\varepsilon>0,((\bar{u}, \bar{v}), \bar{h}) \in\left(W_{T} \cap L^{\infty}\left(Q_{T}\right)\right)^{2} \times\left(\bigcap_{[2,+\infty)} L^{p}\left(Q_{T}\right)\right)$ such that

$$
\begin{cases}\partial_{t} \bar{u}-\Delta \bar{u}=f_{1}(\bar{u}, \bar{v})+\bar{h} 1_{\omega} & \text { in }(0, T) \times \Omega, \\ \partial_{t} \bar{v}-\Delta \bar{v}=f_{2}(\bar{u}, \bar{v}) & \text { in }(0, T) \times \Omega, \\ \bar{u}, \bar{v}=0 & \text { on }(0, T) \times \partial \Omega, \\ (\bar{u}, \bar{v})(0, .)=(0,0),(\bar{u}, \bar{v})(T, .)=(0,0) & \text { in } \Omega,\end{cases}
$$

and

$$
\forall(t, x) \in(T / 8,3 T / 8) \times \omega_{0}, \bar{u}(t, x) \geq \varepsilon .
$$

We construct the reference trajectory $((\bar{u}, \bar{v}), \bar{h})$ on $(0, T / 2)$ to guarantee $(127)$ according to the following statement.

Proposition 5.2. There exists $\varepsilon_{0}>0$ such that for every $0<\varepsilon<\varepsilon_{0}$, there exists $\left(\left(\overline{u_{1}}, \overline{v_{1}}\right), \overline{h_{1}}\right) \in L^{\infty}((0, T / 2) \times \Omega)^{2} \times L^{\infty}((0, T / 2) \times \Omega)$ satisfying

$$
\begin{cases}\partial_{t} \overline{u_{1}}-\Delta \overline{u_{1}}=f_{1}\left(\overline{u_{1}}, \overline{v_{1}}\right)+\overline{h_{1}} 1_{\omega} & \text { in }(0, T / 2) \times \Omega, \\ \partial_{t} \overline{v_{1}}-\Delta \overline{v_{1}}=f_{2}\left(\overline{u_{1}}, \overline{v_{1}}\right) & \text { in }(0, T / 2) \times \Omega, \\ \overline{u_{1}}, \overline{v_{1}}=0 & \text { on }(0, T / 2) \times \partial \Omega, \\ \left(\overline{u_{1}}, \overline{v_{1}}\right)(0, .)=(0,0) & \text { in } \Omega,\end{cases}
$$

and

$$
\begin{gathered}
\forall(t, x) \in(T / 8,3 T / 8) \times \omega_{0}, \overline{u_{1}}(t, x) \geq \varepsilon, \\
\left\|\overline{u_{1}}\right\|_{L^{\infty}((0, T / 2) \times \Omega)} \leq 2 \varepsilon \\
\left\|\overline{v_{1}}(T / 2, .)\right\|_{L^{\infty}(\Omega)} \leq C \varepsilon^{2 k+1} .
\end{gathered}
$$

Proof. Let $\varepsilon>0, \overline{u_{1}} \in C^{\infty}(\overline{(0, T / 2) \times \Omega})$ such that $\operatorname{supp}\left(\overline{u_{1}}\right) \subset \subset(0, T / 2) \times \omega,(129)$ and (130) holds. By a standard Banach fixed point argument, (14) and (130), for $\varepsilon>0$ small enough, there exists a unique solution $\overline{v_{1}} \in L^{\infty}((0, T / 2) \times \Omega)$ of

$$
\begin{cases}\partial_{t} \overline{v_{1}}-\Delta \overline{v_{1}}=f_{2}\left(\overline{u_{1}}, \overline{v_{1}}\right) & \text { in }(0, T / 2) \times \Omega, \\ \overline{v_{1}}=0 & \text { on }(0, T / 2) \times \partial \Omega, \\ \overline{v_{1}}(0, .)=0 & \text { in } \Omega,\end{cases}
$$

in the sense of Definition-Proposition 2.4. From (130), (14), (132) and DefinitionProposition 2.3 (see (7)), we have (131). Finally, we define $\overline{h_{1}} \in L^{\infty}((0, T / 2) \times \Omega)$ thanks to the property of $\operatorname{supp}\left(\overline{u_{1}}\right)$ and (13) (note that $f_{1}(0,)=$.0 ),

$$
\overline{h_{1}}:=\partial_{t} \overline{u_{1}}-\Delta \overline{u_{1}}-f_{1}\left(\overline{u_{1}}, \overline{v_{1}}\right),
$$

which is supported on $(0, T / 2) \times \omega$. This ends the proof of Proposition 5.2.

We construct the reference trajectory $((\bar{u}, \bar{v}), \bar{h})$ of Proposition 5.1 on $(T / 2, T)$ to guarantee $(\bar{u}, \bar{v})(T,)=$.0 according to the following statement, which relies on Proposition 3.7 and the local invertibility of $g_{1}^{1 /(2 k+1)}$. 
Proposition 5.3. Let $\varepsilon_{0}$ be as in Proposition 5.2.

There exists $\varepsilon_{0}^{\prime} \in\left(0, \varepsilon_{0}\right)$ such that, for every $\varepsilon \in\left(0, \varepsilon_{0}^{\prime}\right)$, there exists $\left(\left(\overline{u_{2}}, \overline{v_{2}}\right), \overline{h_{2}}\right) \in$ $L^{\infty}((T / 2, T) \times \Omega)^{2} \times \cap_{[2,+\infty)} L^{p}((T / 2, T) \times \Omega)$ satisfying

$(134)$

$$
\begin{cases}\partial_{t} \overline{u_{2}}-\Delta \overline{u_{2}}=f_{1}\left(\overline{u_{2}}, \overline{v_{2}}\right)+\overline{h_{2}} 1_{\omega} & \text { in }(T / 2, T) \times \Omega, \\ \partial_{t} \overline{v_{2}}-\Delta \overline{v_{2}}=f_{2}\left(\overline{u_{2}}, \overline{v_{2}}\right) & \text { in }(T / 2, T) \times \Omega, \\ \overline{u_{2}}, \overline{v_{2}}=0 & \text { on }(0, T / 2) \times \partial \Omega, \\ \left(\overline{u_{2}}, \overline{v_{2}}\right)(T / 2, .)=\left(0, \overline{v_{1}}(T / 2, .)\right), \quad\left(\overline{u_{2}}, \overline{v_{2}}\right)(T, .)=(0,0) & \text { in } \Omega,\end{cases}
$$

where $\left(\left(\overline{u_{1}}, \overline{v_{1}}\right), \overline{h_{1}}\right)$ is given by Proposition 5.2.

Proof. We recall that $f_{2}(u, v)=g_{1}(u) g_{2}(v)($ see $(14))$.

Step 1: We prove the existence of $a, \alpha, \beta>0$ and a $C^{\infty}$-diffeomorphism $\widetilde{g_{1}}$ : $(-a, a) \rightarrow(-\alpha, \beta)$ such that

$$
\forall x \in(-a, a), g_{1}(x):=\widetilde{g_{1}}(x)^{2 k+1}, g_{2}(x) \neq 0 .
$$

From (14) and the Taylor formula, the map

$$
\widetilde{g_{1}}(x):=\left(\int_{0}^{1} \frac{(1-u)^{2 k}}{(2 k) !} g_{1}^{(2 k+1)}(u x) d u\right)^{1 /(2 k+1)} x,
$$

satisfies $g_{1}(x)=\widetilde{g_{1}}(x)^{2 k+1}$ for every $x \in \mathbb{R}$. Taking into account that

$$
\left.{\widetilde{g_{1}}}^{\prime}(0)=\frac{1}{(2 k+1) !} g_{1}^{(2 k+1)}(0) \neq 0 \text { and } g_{2}(0) \neq 0 \text { (see }(14)\right),
$$

there exists $a>0$ such that $\widetilde{g_{1}} \in C^{\infty}((-a, a) ; \mathbb{R})$, and ${\widetilde{g_{1}}}^{\prime}(x) \neq 0, g_{2}(x) \neq 0$ for every $x \in(-a, a)$.

We conclude from Step 1 that $f_{2}(u, v)={\widetilde{g_{1}}}^{2 k+1}(u) g_{2}(v)$ locally around 0 .

Step 2: Let $0<\varepsilon<\varepsilon_{0}$ be a small parameter which will be fixed later. Let $\left(\left(\overline{u_{1}}, \overline{v_{1}}\right), \overline{h_{1}}\right)$ be as in Proposition 5.2.

We apply Proposition 3.7 with $(0, \tau) \leftarrow(T / 2, T), y_{0} \leftarrow \overline{v_{1}}(T / 2,.) \in L^{\infty}(\Omega)$. From (131), there exists a control $H \in L^{\infty}((T / 2, T) \times \Omega)$ such that

$$
\begin{aligned}
& \left\|H^{\frac{1}{2 k+1}}\right\|_{L^{\infty}((T / 2, T) \times \Omega)} \leq C\left\|\overline{v_{1}}(T / 2, .)\right\|_{L^{\infty}(\Omega)}^{1 /(2 k+1)} \leq C \varepsilon, \\
& H^{\frac{1}{2 k+1}} \in \cap_{p \in[2,+\infty)} X_{(T / 2, T), p}, \quad \text { (see Definition 3.6), } \\
& H(T / 2, .)=H(T, .)=0, \\
& \forall t \in[T / 2, T], \operatorname{supp}(H(t, .)) \subset \subset \omega,
\end{aligned}
$$

and the solution $\overline{v_{2}}$ of

$$
\begin{cases}\partial_{t} \overline{v_{2}}-\Delta \overline{v_{2}}=H & \text { in }(T / 2, T) \times \Omega, \\ \overline{v_{2}}=0 & \text { on }(T / 2, T) \times \partial \Omega, \\ \overline{v_{2}}(T / 2, .)=\overline{v_{1}}(T / 2, .) & \text { in } \Omega\end{cases}
$$

satisfies

$$
\overline{v_{2}}(T, .)=0 .
$$

From (136), (140) and Definition-Proposition 2.3, we have

$$
\left\|\overline{v_{2}}\right\|_{L^{\infty}((T / 2, T) \times \Omega)} \leq C \varepsilon^{2 k+1} .
$$

Moreover, $\overline{v_{2}}$ is the restriction on $(T / 2, T)$ of $\bar{v}$ defined by

$$
\begin{cases}\partial_{t} \bar{v}-\Delta \bar{v}=f_{2}\left(\overline{u_{1}}, \overline{v_{1}}\right) 1_{(0, T / 2)}+H 1_{(T / 2, T)} & \text { in }(0, T) \times \Omega, \\ \bar{v}=0 & \text { on }(0, T) \times \partial \Omega, \\ \bar{v}(0, .)=0 & \text { in } \Omega\end{cases}
$$


Then, by using Proposition 5.2: $\left(\overline{u_{1}}, \overline{v_{1}}\right) \in L^{\infty}\left(Q_{T}\right)^{2}$, (136), (143), Definition 3.6 and Proposition 4.1, we have

$$
\overline{v_{2}} \in \cap_{p \in[2,+\infty)} X_{(T / 2, T), p} .
$$

From (142), for $\varepsilon$ sufficiently small, we have

$$
\left\|\overline{v_{2}}\right\|_{L^{\infty}((T / 2, T) \times \Omega)}<a / 2,
$$

where $a$ is defined in Step 1. Therefore, from (145) and (135), $g_{2}\left(\overline{v_{2}}\right)^{-\frac{1}{2 k+1}}$ is welldefined. Moreover, from (136), for $\varepsilon$ sufficiently small, we have

$$
\left\|H^{\frac{1}{2 k+1}} g_{2}\left(\overline{v_{2}}\right)^{-\frac{1}{2 k+1}}\right\|_{L^{\infty}((0, T / 2) \times \Omega)}<\max (\alpha / 2, \beta / 2),
$$

where $\alpha$ and $\beta$ are defined in Step 1.

Then, we set

$$
\overline{u_{2}}:={\widetilde{g_{1}}}^{-1}\left(H^{\frac{1}{2 k+1}} g_{2}\left(\overline{v_{2}}\right)^{-\frac{1}{2 k+1}}\right) \in L^{\infty}((T / 2, T) \times \Omega),
$$

where $\widetilde{g_{1}}$ is defined as in Step 1. From the fact that $g_{2}^{-\frac{1}{2 k+1}} \in W^{2, \infty}(-a / 2, a / 2)$ (see (135)), (145) and (144), we check that

$$
g_{2}\left(\overline{v_{2}}\right)^{-\frac{1}{2 k+1}} \in \cap_{p \in[2,+\infty)} X_{(T / 2, T), p} .
$$

Taking into account that ${\widetilde{g_{1}}}^{-1} \in W^{2, \infty}(-\alpha / 2, \beta / 2),(137),(148)$ and (147), we verify that

$$
\overline{u_{2}} \in \cap_{p \in[2,+\infty)} X_{(T / 2, T), p} .
$$

Finally, we define $\overline{h_{2}}$ thanks to (147) and (149)

$$
\overline{h_{2}}:=\partial_{t} \overline{u_{2}}-\Delta \overline{u_{2}}-f_{1}\left(\overline{u_{2}}, \overline{v_{2}}\right) \in \cap_{p \in[2,+\infty)} L^{p}((T / 2, T) \times \Omega) .
$$

which is supported on $(T / 2, T) \times \omega$ by $(139)$ and $(13)$ (note that $\left.f_{1}(0,)=0.\right)$. This ends the proof of Proposition 5.3.

\section{Some Generalizations of the GLOBAl NUlL-CONTROLlability FOR "OdD POWER SYSTEMS"}

In this section, we generalize Theorem 2.5 to other parabolic systems. We omit the proofs because in each case, it is a slight adaptation of the strategy of Section 3.

6.1. Linear parabolic operators. We present a natural generalization of the global null-controllability of (Odd) to more general linear parabolic operators than $\partial_{t}-\Delta$.

Proposition 6.1. Let $k \in \mathbb{N}^{*},\left(d_{1}, d_{2}\right) \in(0,+\infty)^{2},\left(b_{1}, b_{2}\right) \in\left(L^{\infty}\left(Q_{T}\right)^{N}\right)^{2},\left(a_{1}, a_{2}\right) \in$ $L^{\infty}\left(Q_{T}\right)^{2}$. Then,

$$
\begin{cases}\partial_{t} u-d_{1} \Delta u+b_{1} \cdot \nabla u+a_{1} u=h 1_{\omega} & \text { in }(0, T) \times \Omega, \\ \partial_{t} v-d_{2} \Delta v+b_{2} . \nabla v+a_{2} v=u^{2 k+1} & \text { in }(0, T) \times \Omega, \\ u, v=0 & \text { on }(0, T) \times \partial \Omega, \\ (u, v)(0, .)=\left(u_{0}, v_{0}\right) & \text { in } \Omega,\end{cases}
$$

is globally null-controllable.

The proof is based on a Carleman estimate different from the one in Proposition 4.3 which can be found in [18, Lemma 2.1]. 
6.2. Global null-controllability result for particular superlinearities. We state a global null-controllability result linked with the global null-controllability of the semilinear heat equation.

Proposition 6.2. Let $k \in \mathbb{N}^{*}, f \in C^{\infty}(\mathbb{R} ; \mathbb{R})$ such that

$$
f(0)=0 \text { and } \frac{f(s)}{s \log ^{3 / 2}(1+|s|)} \rightarrow 0 \text { when }|s| \rightarrow+\infty .
$$

Then,

$$
\begin{cases}\partial_{t} u-\Delta u=f(u)+h 1_{\omega} & \text { in }(0, T) \times \Omega, \\ \partial_{t} v-\Delta v=u^{2 k+1} & \text { in }(0, T) \times \Omega, \\ u, v=0 & \text { on }(0, T) \times \partial \Omega, \\ (u, v)(0, .)=\left(u_{0}, v_{0}\right) & \text { in } \Omega,\end{cases}
$$

is globally null-controllable.

The proof is based on the global null-controllability of the semilinear heat equation with superlinearity as the function $f$. Particularly, we can see [19, Theorem 1.2] proved by Enrique Fernandez-Cara and Enrique Zuazua or [18, Theorem 1.7].

6.3. Global null-controllability for all "power systems". Let $n \in \mathbb{N}^{*}$. We have seen that (Power), with $n$ an even integer, is not (globally) null-controllable by the maximum principle (see Proposition 1.9) but (Power), with $n$ an odd integer, is globally null-controllable (see Theorem 2.5). In this section, we consider the following system:

(PowerO)

$$
\begin{cases}\partial_{t} u-\Delta u=h 1_{\omega} & \text { in }(0, T) \times \Omega, \\ \partial_{t} v-\Delta v=|u|^{n-1} u & \text { in }(0, T) \times \Omega, \\ u, v=0 & \text { on }(0, T) \times \partial \Omega, \\ (u, v)(0, .)=\left(u_{0}, v_{0}\right) & \text { in } \Omega\end{cases}
$$

Definition 6.3. The mapping $\Phi_{n}: x \in \mathbb{R} \mapsto|x|^{n-1} x \in \mathbb{R}$ is one-to-one. We note its inverse function $\Phi_{n}^{-1}$.

Remark 6.4. For $n$ an even integer, $\Phi_{n}(u)=u^{n}$ if $u \geq 0$ and $\Phi_{n}(u)=-u^{n}$ if $u<0$. Whereas for $n$ an odd power, $\Phi_{n}(u)=u^{n}$ for every $u \in \mathbb{R}$.

We have a generalization of Theorem 2.5.

Theorem 6.5. The system (PowerO) is globally null-controllable (in the sense of Definition 1.4).

More precisely, there exists $\left(C_{p}\right)_{p \in[2,+\infty)} \in(0, \infty)^{[2,+\infty)}$ such that for every initial data $\left(u_{0}, v_{0}\right) \in L^{\infty}(\Omega)^{2}$, there exists a control $h \in \bigcap_{p \in[2,+\infty)} L^{p}\left(Q_{T}\right)$ satisfying

$$
\forall p \in[2,+\infty),\|h\|_{L^{p}\left(Q_{T}\right)} \leq C_{p}\left(\left\|u_{0}\right\|_{L^{\infty}(\Omega)}+\left\|v_{0}\right\|_{L^{\infty}(\Omega)}^{1 / n}\right),
$$

and the solution $(u, v)$ of (PowerO) verifies

$$
(u, v)(T, .)=(0,0) .
$$

The strategy of proof of Theorem 6.5 is the same as for Theorem 2.5. It is based on the following key result. 
Proposition 6.6. For every $\tau>0$, there exists $C_{\tau}>0$ such that for every $y_{0} \in L^{\infty}(\Omega)$, there exists a control $h_{\tau} \in L^{\infty}\left(Q_{\tau}\right)$ which verifies

$$
\begin{aligned}
& \left\|\Phi_{n}^{-1}\left(h_{\tau}\right)\right\|_{L^{\infty}\left(Q_{\tau}\right)} \leq C_{\tau}\left\|y_{0}\right\|_{L^{\infty}(\Omega)}^{1 / n}, \\
& \Phi_{n}^{-1}\left(h_{\tau}\right) \in \bigcap_{p \in[2,+\infty)} X_{\tau, p}, \\
& \forall p \in[2,+\infty), \exists C_{\tau, p}>0,\left\|\Phi_{n}^{-1}\left(h_{\tau}\right)\right\|_{X_{\tau, p}} \leq C_{\tau, p}\left\|y_{0}\right\|_{L^{\infty}(\Omega)}^{1 / n}, \\
& h_{\tau}(0, .)=h_{\tau}(\tau, .)=0, \\
& \forall t \in[0, \tau], \operatorname{supp}\left(h_{\tau}(t, .)\right) \subset \subset \omega,
\end{aligned}
$$

such that the solution $y \in L^{\infty}\left(Q_{\tau}\right)$ of (18) satisfies $y(\tau,)=$.0 .

The proof of Proposition 6.6 is a slight adaptation of Section 4. First, we get a Carleman estimate in $L^{n+1}$ (see Theorem 4.4). Secondly, we use a penalized duality method in $L^{(n+1) / n}$ as in Section 4.2 taking into account that the antiderivative of the continuous mapping $\Phi_{n}^{-1}$ (see Definition 6.3) is the strictly convex function

$$
x \in \mathbb{R} \mapsto \frac{n}{n+1}|x|^{\frac{n+1}{n}}:=\left\{\begin{array}{cl}
\frac{n}{n+1} \exp \left(\frac{n+1}{n} \log (|x|)\right) & \text { if } x \neq 0, \\
0 & \text { if } x=0 .
\end{array}\right.
$$

6.4. Global null-controllability for "even power systems" in $\mathbb{C}$. Let $k \in \mathbb{N}^{*}$. We have seen in Proposition 1.9 that global null-controllability does not hold for

(Even)

$$
\begin{cases}\partial_{t} u-\Delta u=h 1_{\omega} & \text { in }(0, T) \times \Omega, \\ \partial_{t} v-\Delta v=u^{2 k} & \text { in }(0, T) \times \Omega, \\ u, v=0 & \text { on }(0, T) \times \partial \Omega, \\ (u, v)(0, .)=\left(u_{0}, v_{0}\right) & \text { in } \Omega .\end{cases}
$$

A natural question, asked by Luc Robbiano, is: what happens if we consider complexvalued functions? A positive answer i.e. a global null-controllability result for (Even) with $k=1$ is given in [11] (see [11, Theorem 3]). Here, we want to generalize this result for every $k \in \mathbb{N}^{*}$. We have the following result.

Theorem 6.7. The system (Even) is globally null-controllable (in the sense of Definition 1.4).

More precisely, there exists $\left(C_{p}\right)_{p \in[2,+\infty)} \in(0, \infty)^{[2,+\infty)}$ such that for every initial data $\left(u_{0}, v_{0}\right) \in L^{\infty}(\Omega)^{2}$, there exists a control $h \in \bigcap_{p \in[2,+\infty)} L^{p}\left(Q_{T} ; \mathbb{C}\right)$ satisfying

$$
\forall p \in[2,+\infty),\|h\|_{L^{p}\left(Q_{T} ; \mathbb{C}\right)} \leq C_{p}\left(\left\|u_{0}\right\|_{L^{\infty}(\Omega)}+\left\|v_{0}\right\|_{L^{\infty}(\Omega)}^{1 /(2 k)}\right),
$$

and the solution $(u, v) \in L^{\infty}\left(Q_{T}, \mathbb{C}\right)^{2}$ of (Even) verifies

$$
(u, v)(T, .)=(0,0) .
$$

The strategy of proof of Theorem 6.7 is the same as for Theorem 2.5 (see Section 3). The first step of the proof i.e. Section 3.1 does not change but we have to modify some arguments given in Section 3.2.

Let us fix $\left(u_{0}, v_{0}\right) \in L^{\infty}(\Omega)^{2}$ until the end of the section.

6.4.1. First step of the proof: steer $u$ to 0. First, we find a control of (Even) which steers $u$ to 0 in time $T / 2$ (see the proof of Proposition 3.1).

Proposition 6.8. There exists $h_{1} \in L^{\infty}((0, T / 2) \times \Omega ; \mathbb{R})$ satisfying

$$
\left\|h_{1}\right\|_{L^{\infty}((0, T / 2) \times \Omega ; \mathbb{R})} \leq C\left\|u_{0}\right\|_{L^{\infty}(\Omega)},
$$


such that the solution $\left(u_{1}, v_{1}\right) \in L^{\infty}((0, T / 2) \times \Omega ; \mathbb{R})^{2}$ of

$$
\begin{cases}\partial_{t} u_{1}-\Delta u_{1}=h_{1} 1_{\omega} & \text { in }(0, T / 2) \times \Omega, \\ \partial_{t} v_{1}-\Delta v_{1}=u_{1}^{2 k} & \text { in }(0, T / 2) \times \Omega, \\ u_{1}, v_{1}=0 & \text { on }(0, T / 2) \times \partial \Omega, \\ \left(u_{1}, v_{1}\right)(0, .)=\left(u_{0}, v_{0}\right) & \text { in } \Omega,\end{cases}
$$

satisfies $u_{1}(T / 2,)=$.0 . Moreover, we have

$$
\left\|v_{1}(T / 2, .)\right\|_{L^{\infty}(\Omega)} \leq C\left(\left\|u_{0}\right\|_{L^{\infty}(\Omega)}^{2 k}+\left\|v_{0}\right\|_{L^{\infty}(\Omega)}\right) .
$$

6.4.2. Second step of the proof: steer $v$ to 0 . The aim of this part is to find a complex control of (Even) which steers $v$ to 0 (and $u$ from 0 to 0 ) in time $T$.

Proposition 6.9. Let $\left(\left(u_{1}, v_{1}\right), h_{1}\right)$ as in Proposition 6.8 .

There exists a control $h_{2} \in \bigcap_{p \in[2,+\infty)} L^{p}((T / 2, T) \times \Omega ; \mathbb{C})$ satisfying

$$
\forall p \in[2,+\infty),\left\|h_{2}\right\|_{\left.L^{p}((T / 2, T) \times \Omega) ; \mathbb{C}\right)} \leq C_{p}\left(\left\|u_{0}\right\|_{L^{\infty}(\Omega)}+\left\|v_{0}\right\|_{L^{\infty}(\Omega)}^{1 /(2 k)}\right) .
$$

such that the solution $\left(u_{2}, v_{2}\right) \in L^{\infty}((T / 2, T) \times \Omega ; \mathbb{C})^{2}$ of

$$
\begin{cases}\partial_{t} u_{2}-\Delta u_{2}=h_{2} 1_{\omega} & \text { in }(T / 2, T) \times \Omega, \\ \partial_{t} v_{2}-\Delta v_{2}=u_{2}^{2 k} & \text { in }(T / 2, T) \times \Omega, \\ u_{2}, v_{2}=0 & \text { on }(T / 2, T) \times \partial \Omega, \\ \left(u_{2}, v_{2}\right)(T / 2, .)=\left(0, v_{1}(T / 2, .)\right) & \text { in } \Omega,\end{cases}
$$

satisfies $\left(u_{2}, v_{2}\right)(T,)=.(0,0)$.

Our approach consists in looking at the second equation of (Odd) like a controlled heat equation where the state is $v(t,$.$) and the control input is u^{2 k}(t,$.$) . Here, the$ question consists in proving that the heat equation is null-controllable with a localized control which is as an even power of a regular complex function.

Now, we can prove Proposition 6.9.

Proof. We apply Proposition 6.6 with $n=4 k,(0, \tau) \leftarrow(T / 2, T), y_{0} \leftarrow v_{1}(T / 2,.) \in$ $L^{\infty}(\Omega)$. Then, there exists a control $H \in L^{\infty}((T / 2, T) \times \Omega)$ such that

$$
\begin{aligned}
& \Phi_{4 k}^{-1}(H) \in \bigcap_{p \in[2,+\infty)} X_{(T / 2, T), p} \\
& \forall p \in[2,+\infty),\left\|\Phi_{4 k}^{-1}(H)\right\|_{X_{(T / 2, T), p}} \leq C_{p}\left\|v_{1}(T / 2, .)\right\|_{L^{\infty}(\Omega)}^{1 /(4 k)}, \\
& H(T / 2, .)=H(T, .)=0, \\
& \forall t \in[T / 2, T], \operatorname{supp}(H(t, .)) \subset \subset \omega,
\end{aligned}
$$

and the solution $v_{2}$ of

$$
\begin{cases}\partial_{t} v_{2}-\Delta v_{2}=H & \text { in }(T / 2, T) \times \Omega, \\ v_{2}=0 & \text { on }(T / 2, T) \times \partial \Omega, \\ v_{2}(T / 2, .)=v_{1}(T / 2, .) & \text { in } \Omega,\end{cases}
$$

satisfies

$$
v_{2}(T, .)=0
$$

We introduce the notation

$$
\alpha:=e^{\frac{i \pi}{2 k}} \in \mathbb{C} .
$$

We take $u_{2}$, the complex-valued function, as

$$
u_{2}:=\left(\left(\left(\Phi_{4 k}^{-1}(H)\right)^{+}\right)_{26}^{2}+\alpha\left(\left(\left(\Phi_{4 k}^{-1}(H)\right)^{-}\right)^{2},\right.\right.
$$


where the positive and negative parts of a real number $x$ are defined as follows

$$
x^{+}:=\max (x, 0), \quad x^{-}:=-\min (x, 0) .
$$

From Definition 6.3 and (162), we verify that

$$
u^{2 k}=\left(\left(\left(\Phi_{4 k}^{-1}(H)\right)^{+}\right)^{4 k}+\alpha^{2 k}\left(\left(\left(\Phi_{4 k}^{-1}(H)\right)^{-}\right)^{4 k}=H^{+}-H^{-}=H .\right.\right.
$$

Moreover, we have

$$
x \mapsto\left(x^{+}\right)^{2} \in W_{\text {loc }}^{2, \infty}(\mathbb{R}), \quad x \mapsto\left(x^{-}\right)^{2} \in W_{\text {loc }}^{2, \infty}(\mathbb{R}) .
$$

From (156), (162) and (164), we have

$$
u_{2} \in\left(\bigcap_{p \in[2,+\infty)} X_{(T / 2, T), p}\right) \cap L^{\infty}((T / 2, T) \times \Omega ; \mathbb{C}) .
$$

We have, from (158),

$$
u_{2}(T / 2, .)=u_{2}(T, .)=0 .
$$

Then, we set, from (162), (165) and (159),

$$
h_{2}:=\partial_{t} u_{2}-\Delta u_{2} \in \bigcap_{p \in[2,+\infty)} L^{p}((T / 2, T) \times \Omega ; \mathbb{C}),
$$

which is supported in $(T / 2, T) \times \omega$. From (157), (154) and (162), we have

$$
\forall p \in[2,+\infty),\left\|h_{2}\right\|_{\left.L^{p}((T / 2, T) \times \Omega) ; \mathbb{C}\right)} \leq C_{p}\left(\left\|u_{0}\right\|_{L^{\infty}(\Omega)}+\left\|v_{0}\right\|_{L^{\infty}(\Omega)}^{1 /(2 k)}\right) .
$$

By using (159), (160), (161), (163), (165), (166), (167) and (168), we check that $\left(\left(u_{2}, v_{2}\right), h_{2}\right)$ satisfies Proposition 6.9.

\section{Appendix A.}

\section{A.1. Proof of the uniqueness of the point 2. of Definition-Proposition 2.4.}

Proof. Let $\left(u_{0}, v_{0}\right) \in L^{\infty}(\Omega)^{2}, h \in L^{2}\left(Q_{T}\right)$.

Let $(u, v) \in\left(W_{T} \cap L^{\infty}\left(Q_{T}\right)\right)^{2}$ and $(\widetilde{u}, \widetilde{v}) \in\left(W_{T} \cap L^{\infty}\left(Q_{T}\right)\right)^{2}$ be two solutions of (NL). Then, the function $(\widehat{u}, \widehat{v}):=(u-\widetilde{u}, v-\widetilde{v}) \in\left(W_{T} \cap L^{\infty}\left(Q_{T}\right)\right)^{2}$ satisfies (in the weak sense)

$$
\begin{cases}\partial_{t} \widehat{u}-\Delta \widehat{u}=f_{1}(u, v)-f_{1}(\widetilde{u}, \widetilde{v}) & \text { in }(0, T) \times \Omega, \\ \partial_{t} \widehat{v}-\Delta \widehat{v}=f_{2}(u, v)-f_{2}(\widetilde{u}, \widetilde{v}) & \text { in }(0, T) \times \Omega, \\ \widehat{u}, \widehat{v}=0 & \text { on }(0, T) \times \partial \Omega \\ (\widehat{u}, \widehat{v})(0, .)=(0,0) & \text { in } \Omega\end{cases}
$$

By taking $\left(w_{1}, w_{2}\right):=(\widehat{u}, \widehat{v})$ in the variational formulation of (169) (see (8) and (9)) and by using the fact that the mapping $t \mapsto\left\|(\widehat{u}(t), \widehat{v}(t))^{T}\right\|_{L^{2}(\Omega)^{2}}^{2}$ is absolutely continuous (see [16, Section 5.9.2, Theorem 3]) with for a.e. $0 \leq t \leq T$,

$$
\frac{d}{d t}\left\|(\widehat{u}(t), \widehat{v}(t))^{T}\right\|_{L^{2}(\Omega)^{2}}^{2}=2\left(\left(\left(\partial_{t} \widehat{u}(t), \widehat{u}(t)\right),\left(\partial_{t} \widehat{v}(t), \widehat{v}(t)\right)\right)^{T}\right)_{\left(H^{-1}(\Omega)^{2}, H_{0}^{1}(\Omega)^{2}\right)},
$$

we find that for a.e. $0 \leq t \leq T$,

$$
\begin{aligned}
& \frac{1}{2} \frac{d}{d t}\left(\left\|(\widehat{u}(t), \widehat{v}(t))^{T}\right\|_{L^{2}(\Omega)^{2}}^{2}\right)+\left\|(\nabla \widehat{u}, \nabla \widehat{v})^{T}\right\|_{L^{2}(\Omega)^{2}}^{2} \\
& =\left(\left(\left(f_{1}(u, v)-f_{1}(\widetilde{u}, \widetilde{v}), \widehat{u}\right),\left(f_{2}(u, v)-f_{2}(\widetilde{u}, \widetilde{v}), \widehat{v}\right)\right)^{T}\right)_{\left(L^{2}(\Omega)^{2}, L^{2}(\Omega)^{2}\right)} .
\end{aligned}
$$

By using the facts that $(u, \widetilde{u}) \in L^{\infty}\left(Q_{T}\right)^{2}$ and $(x, y) \mapsto f_{1}(x, y),(x, y) \mapsto f_{2}(x, y)$ are locally Lipschitz on $\mathbb{R}^{2}$, we find the differential inequality

$$
\frac{d}{d t}\left(\left\|(\widehat{u}(t), \widehat{v}(t))^{T}\right\|_{L^{2}(\Omega)^{2}}^{2}\right) \leq C\left(\underset{27}{\left.\left\|(\widehat{u}(t), \widehat{v}(t))^{T}\right\|_{L^{2}(\Omega)^{2}}^{2}\right)} \text { for a.e. } 0 \leq t \leq T .\right.
$$


Gronwall lemma and the initial condition $(\widehat{u}(0), \widehat{v}(0))=(0,0)$ (see $(10))$ prove that $(\widehat{u}(t), \widehat{v}(t))=(0,0)$. Consequently, $(u, v)=(\widetilde{u}, \widetilde{v})$.

\section{A.2. Proof of the general case for Corollary 4.6.}

Proof. Let $\varphi_{T} \in L^{2 k+2}(\Omega)$ and $\left(\varphi_{T, n}\right)_{n \in \mathbb{N}} \in\left(C_{0}^{\infty}(\Omega)\right)^{\mathbb{N}}$ such that

$$
\varphi_{T, n} \underset{n \rightarrow+\infty}{\rightarrow} \varphi_{T} \text { in } L^{2 k+2}(\Omega) .
$$

We denote by $\left(\varphi_{n}\right)_{n \in \mathbb{N}}$ the sequence of solutions of

$$
\begin{cases}-\partial_{t} \varphi_{n}-\Delta \varphi_{n}=0 & (0, T) \times \Omega \\ \varphi_{n}=0 & (0, T) \times \partial \Omega \\ \varphi_{n}(T, .)=\varphi_{T, n} & \Omega\end{cases}
$$

The estimates (66) and (65) hold true for $\left(\varphi_{n}\right)_{n \in \mathbb{N}}$ by the Step 1 of the proof of Corollary 4.6. Moreover, from (170), (171), (36) and Definition-Proposition 2.3 (particularly $(7)$ for $p=2 k+2$ ), we have

$$
\left\|\varphi_{n}-\varphi\right\|_{L^{2 k+2}\left(Q_{T}\right)} \leq C\left\|\varphi_{T, n}-\varphi_{T}\right\|_{L^{2 k+2}(\Omega)} \underset{n \rightarrow+\infty}{\rightarrow} 0,
$$

where $\varphi \in L^{2 k+2}\left(Q_{T}\right)$ is the solution of (36). By using

$$
\chi^{2 k+2} e^{-(k+1) s \rho(x) \eta(t)}(s \eta)^{3(k+1)} \in L^{\infty}\left(Q_{T}\right),
$$

and (172), we get

$$
\begin{aligned}
& \iint_{(0, T) \times \omega} \chi^{2 k+2} e^{-(k+1) s \rho \eta}(s \eta)^{3(k+1)}\left|\varphi_{n}\right|^{2 k+2} \\
& \underset{n \rightarrow+\infty}{\rightarrow} \iint_{(0, T) \times \omega} \chi^{2 k+2} e^{-(k+1) s \rho \eta}(s \eta)^{3(k+1)}|\varphi|^{2 k+2} .
\end{aligned}
$$

From (66), (65) applied to $\left(\varphi_{n}\right)_{n \in \mathbb{N}}$ and $(174)$, we deduce that $\left(\varphi_{n}(0, .)\right)_{n \in \mathbb{N}}$, (respectively $\left(e^{-\rho s \eta / 2}(s \eta)^{-m / 2} \varphi_{n}\right)_{n \in \mathbb{N}}$, respectively $\left.\left(e^{-\rho s \eta / 2}(s \eta)^{-(m+2) / 2} \nabla \varphi_{n}\right)_{n \in \mathbb{N}}\right)$ is bounded in $L^{2 k+2}(\Omega)$, (respectively $L^{2 k+2}\left(Q_{T}\right)$, respectively $L^{2 k+2}\left(Q_{T}\right)$ ) which is a Banach reflexive space. Then, up to a subsequence, we can assume that

$$
\begin{gathered}
\varphi_{n}(0, .) \underset{n \rightarrow+\infty}{\rightarrow} \varphi(0, .) \text { in } L^{2 k+2}(\Omega), \\
e^{-\rho s \eta / 2}(s \eta)^{-m / 2} \varphi_{n} \underset{n \rightarrow+\infty}{\rightarrow} e^{-\rho s \eta / 2}(s \eta)^{-m / 2} \varphi \text { in } L^{2 k+2}\left(Q_{T}\right), \\
e^{-\rho s \eta / 2}(s \eta)^{-(m+2) / 2} \nabla \varphi_{n} \underset{n \rightarrow+\infty}{\rightarrow} e^{-\rho s \eta / 2}(s \eta)^{-(m+2) / 2} \nabla \varphi \text { in } L^{2 k+2}\left(Q_{T}\right) .
\end{gathered}
$$

In particular, we have

$$
\begin{aligned}
\|\varphi(0, .)\|_{L^{2 k+2}(\Omega)}^{2 k+2} & \leq \liminf _{n \rightarrow+\infty}\left\|\varphi_{n}(0, .)\right\|_{L^{2 k+2}(\Omega)}^{2 k+2} \\
& \leq C_{s} \liminf _{n \rightarrow+\infty} \iint_{(0, T) \times \omega} \chi^{2 k+2} e^{-(k+1) s \rho(x) \eta(t)}(s \eta)^{3(k+1)}\left|\varphi_{n}\right|^{2 k+2} d x d t \\
& \leq C_{s} \iint_{(0, T) \times \omega} \chi^{2 k+2} e^{-(k+1) s \rho(x) \eta(t)}(s \eta)^{3(k+1)}|\varphi|^{2 k+2} d x d t,
\end{aligned}
$$


and

(179)

$$
\begin{aligned}
& \iint_{(0, T) \times \Omega} e^{-(k+1) s \rho \eta}\left((s \eta)^{-(k+1) m}|\varphi|^{2 k+2}+(s \eta)^{-(k+1)(m+2)}|\nabla \varphi|^{2 k+2}\right) \\
& \leq \liminf _{n \rightarrow+\infty} \iint_{(0, T) \times \Omega} e^{-(k+1) s \rho \eta}\left((s \eta)^{-(k+1) m}\left|\varphi_{n}\right|^{2 k+2}+(s \eta)^{-(k+1)(m+2)}\left|\nabla \varphi_{n}\right|^{2 k+2}\right) \\
& \leq C \liminf _{n \rightarrow+\infty} \iint_{(0, T) \times \omega} \chi^{2 k+2} e^{-(k+1) s \rho \eta}(s \eta)^{3(k+1)}\left|\varphi_{n}\right|^{2 k+2} \\
& \leq C \iint_{(0, T) \times \omega} \chi^{2 k+2} e^{-(k+1) s \rho \eta}(s \eta)^{3(k+1)}|\varphi|^{2 k+2} .
\end{aligned}
$$

The estimates (178) and (179) conclude the proof.

\section{REFERENCES}

[1] Farid Ammar Khodja, Assia Benabdallah, and Cédric Dupaix. Null-controllability of some reaction-diffusion systems with one control force. J. Math. Anal. Appl., 320(2):928-943, 2006.

[2] Farid Ammar Khodja, Assia Benabdallah, Cédrix Dupaix, and Manuel González-Burgos. A generalization of the Kalman rank condition for time-dependent coupled linear parabolic systems. Differ. Equ. Appl., 1(3):427-457, 2009.

[3] Farid Ammar-Khodja, Assia Benabdallah, Manuel González-Burgos, and Luz de Teresa. Recent results on the controllability of linear coupled parabolic problems: a survey. Math. Control Relat. Fields, 1(3):267-306, 2011.

[4] Viorel Barbu. Exact controllability of the superlinear heat equation. Appl. Math. Optim., $42(1): 73-89,2000$.

[5] Claude Bardos and Luc Tartar. Sur l'unicité rétrograde des équations paraboliques et quelques questions voisines. Arch. Rational Mech. Anal., 50:10-25, 1973.

[6] Olivier Bodart, Manuel González-Burgos, and Rosario Pérez-Garcí a. Existence of insensitizing controls for a semilinear heat equation with a superlinear nonlinearity. Comm. Partial Differential Equations, 29(7-8):1017-1050, 2004.

[7] Felipe Wallison Chaves-Silva and Sergio Guerrero. A uniform controllability result for the Keller-Segel system. Asymptot. Anal., 92(3-4):313-338, 2015.

[8] Jean-Michel Coron. Global asymptotic stabilization for controllable systems without drift. Math. Control Signals Systems, 5(3):295-312, 1992.

[9] Jean-Michel Coron. Control and nonlinearity, volume 136 of Mathematical Surveys and Monographs. American Mathematical Society, Providence, RI, 2007.

[10] Jean-Michel Coron, Sergio Guerrero, Philippe Martin, and Lionel Rosier. Homogeneity applied to the controllability of a system of parabolic equations. In 2015 European Control Conference (ECC 2015), Proceedings of the 2015 European Control Conference (ECC 2015), pages 24702475, Linz, Austria, July 2015.

[11] Jean-Michel Coron, Sergio Guerrero, and Lionel Rosier. Null controllability of a parabolic system with a cubic coupling term. SIAM J. Control Optim., 48(8):5629-5653, 2010.

[12] Jean-Michel Coron and Jean-Philippe Guilleron. Control of three heat equations with two cubic nonlinearities. SIAM J. Control Optim., To appear in 2016.

[13] Luz de Teresa. Insensitizing controls for a semilinear heat equation. Comm. Partial Differential Equations, 25(1-2):39-72, 2000.

[14] Robert Denk, Matthias Hieber, and Jan Prüss. Optimal $L^{p}-L^{q}$-estimates for parabolic boundary value problems with inhomogeneous data. Math. Z., 257(1):193-224, 2007.

[15] Michel Duprez and Pierre Lissy. Indirect controllability of some linear parabolic systems of $m$ equations with $m-1$ controls involving coupling terms of zero or first order. J. Math. Pures Appl. (9), 106(5):905-934, 2016.

[16] Lawrence C. Evans. Partial differential equations, volume 19 of Graduate Studies in Mathematics. American Mathematical Society, Providence, RI, second edition, 2010.

[17] Enrique Fernández-Cara, Manuel González-Burgos, Sergio Guerrero, and Jean-Pierre Puel. Null controllability of the heat equation with boundary Fourier conditions: the linear case. ESAIM Control Optim. Calc. Var., 12(3):442-465, 2006.

[18] Enrique Fernández-Cara and Sergio Guerrero. Global Carleman inequalities for parabolic systems and applications to controllability. SIAM J. Control Optim., 45(4):1399-1446 (electronic), 2006 . 
[19] Enrique Fernández-Cara and Enrique Zuazua. Null and approximate controllability for weakly blowing up semilinear heat equations. Ann. Inst. H. Poincaré Anal. Non Linéaire, 17(5):583$616,2000$.

[20] Andrei V. Fursikov and Oleg Yu. Imanuvilov. Controllability of evolution equations, volume 34 of Lecture Notes Series. Seoul National University, Research Institute of Mathematics, Global Analysis Research Center, Seoul, 1996.

[21] Manuel González-Burgos and Rosario Pérez-García. Controllability results for some nonlinear coupled parabolic systems by one control force. Asymptot. Anal., 46(2):123-162, 2006.

[22] Olga Aleksandrovna. Ladyzenskaja, Vsevolod Alekseevich Solonnikov, and Nina Nikolaevna Uralceva. Linear and quasilinear equations of parabolic type. Translated from the Russian by S. Smith. Translations of Mathematical Monographs, Vol. 23. American Mathematical Society, Providence, R.I., 1968.

[23] Kévin Le Balc'h. Controllability of a $4 \times 4$ quadratic reaction-diffusion system. ArXiv e-prints, November 2017.

[24] Jérôme Le Rousseau and Gilles Lebeau. On Carleman estimates for elliptic and parabolic operators. Applications to unique continuation and control of parabolic equations. ESAIM Control Optim. Calc. Var., 18(3):712-747, 2012.

[25] Gilles Lebeau and Luc Robbiano. Contrôle exacte de l'équation de la chaleur. In Séminaire sur les Équations aux Dérivées Partielles, 1994-1995, pages Exp. No. VII, 13. École Polytech., Palaiseau, 1995.

[26] Juan Límaco, Marcondes Clark, Alexandro Marinho, Silvado B. de Menezes, and Aldo T. Louredo. Null controllability of some reaction-diffusion systems with only one control force in moving domains. Chin. Ann. Math. Ser. B, 37(1):29-52, 2016.

[27] Jacques-Louis Lions. Exact controllability, stabilization and perturbations for distributed systems. SIAM Rev., 30(1):1-68, 1988.

[28] Jacques Simon. Compact sets in the space $L^{p}(0, T ; B)$. Ann. Mat. Pura Appl. (4), 146:65-96, 1987.

[29] Gensheng Wang and Liang Zhang. Exact local controllability of a one-control reaction-diffusion system. J. Optim. Theory Appl., 131(3):453-467, 2006.

[30] Zhuoqun Wu, Jingxue Yin, and Chunpeng Wang. Elliptic $\&$ parabolic equations. World Scientific Publishing Co. Pte. Ltd., Hackensack, NJ, 2006.

[31] Christophe Zhang. Internal control of systems of semilinear coupled 1-D wave equations. ArXiv e-prints, May 2017.

[32] Enrique Zuazua. Controllability and observability of partial differential equations: some results and open problems. In Handbook of differential equations: evolutionary equations. Vol. III, Handb. Differ. Equ., pages 527-621. Elsevier/North-Holland, Amsterdam, 2007. 